\title{
Immersed Boundary Method with Non-Uniform Distribution of Lagrangian Markers for a Non-Uniform Eulerian Mesh
}

\author{
G. Akiki and S. Balachandar \\ Department of Mechanical and Aerospace Engineering, University of Florida, Gainesville
}

\begin{abstract}
This study presents a technique to incorporate spheres in a channel flow that uses a non-uniform Eulerian grid using immersed boundary methods with direct forcing. An efficient algorithm is presented which distributes the Lagrangian markers non-uniformly to match the fluid grid and keep the number of markers optimized. Also a novel method to calculate the area weights of the Lagrangian markers is given. It is observed that even the best available algorithms for uniform distribution of markers on a sphere results in a finite error. Using vector spherical harmonics, this error is quantified and reduced to machine precision. A series of simulations of a stationary and moving sphere in a periodic channel at Reynolds number range of 1-100 are presented. Results for a sphere in an ambient shear flow in close proximity of a wall are also shown, where the present non-uniform distribution offers an order of magnitude reduction over uniform distribution of Lagrangian markers. Simulations of a random cluster of 640 monodipserse spherical particles show a 77\% reduction in Lagrangian markers with an error of $0.135 \%$ in computing the total drag.
\end{abstract}

Keyword: Immersed-boundary method; Non-uniform grid; Particle channel flow; Direct forcing

\section{Introduction}

Fluid-structure interaction has been an area of active research over the past few decades. Adaptive meshing and arbitrary Lagrangian Eulerian technique (Hirt et. al. 1974) were some of the methods developed to incorporate dynamic or flexible boundaries in a flow. These approaches require expensive remeshing to accommodate the updated position and form of the immersed body. Peskin (1972) then introduced the immersed boundary method (IBM) to resolve flow patterns around heart valves. The IBM presents a relatively simple technique to simulate moving immersed bodies. It has proven to be a powerful technique for simulating deformable fluid-solid interfaces as well. Its appeal lies in its ability to use a fixed Eulerian grid, and it does not require a body-fitted mesh. The fluid-solid interface is represented by Lagrangian markers that move with the body. Then a forcing term on these markers is added to the governing equations to account for the boundary conditions on the interface. Mittal and Iaccarino (2005) presents a review of the various types of IBMs. Their differences lie in the method by which the forcing term is applied. For this study, a direct-forcing method based on Uhlmann (2005) is used.

IBM uses a distribution function to interpolate the fluid velocity from an Eulerian grid onto the Lagrangian markers and to spread the forcing term computed at the Lagrangian markers onto the surrounding Eulerian nodes. Every distribution function should ensure the conservation of the forces and 
torques upon mapping from the Lagrangian markers to the Eulerian grid. One of the more popular distribution function is known as the Dirac Delta Function (DDF), which was originally proposed for a uniformed spaced Eulerian grid by Roma (1999). The DDF has been designed to conserve total force and torque when used with a uniform Eulerian mesh, but the conservation properties do not readily carry over for a non-uniform Eulerian grid. This limitation narrows the capabilities of the IBM in resolving complicated geometries and thin boundary layers, where a non-uniform grid may be desired. As a way around it, several studies, such as Lee et al. (2011), utilized a fine uniform mesh in the region that embeds the immersed boundary and a stretched non-uniform grid away of that vicinity. Zhang et. al. (2004) developed the immersed finite element method that uses the reproducing kernel particle method (RKPM) as a distribution function. This method works for both uniform and non-uniform Eulerian meshes. Later, Pinelli et al. (2010) presented a correction to the DDF rendering the IBM functional with both uniform and non-uniform grids.

Our main interest of this study lies in the incorporation of spherical particles in a channel flow code that uses spectral or higher order spatial discretization. This would provide a tool to investigate problems such as riverbed erosions and porous media. Channel flow codes typically use a non-uniform grid in the wall-normal direction for a higher concentration of Eulerian grid near the wall in order to accurately capture the boundary layer dynamics. This non-uniformity of the Eulerian grid can be accommodated in IBM with the use of the correction to the DDF introduced by Pinelli et al. (2010).

However, there still exists a significant challenge in terms of the distribution of Lagrangian markers. Most studies in the past have used IBM with an even distribution of Lagrangian markers over the interface. In the case of spheres, a perfectly uniform distribution of Lagrangian markers on the surface of the sphere still remains an open mathematical question, but there are algorithms for obtaining a nearly uniform distribution (Saff and Kuijlaars (1997). For a uniform Eulerian grid, Uhlmann (2005) shows that the Lagrangian markers should be spaced such that the surface area associated with each Lagrangian marker must be approximately equal to the square of the Eulerian grid spacing. Any further increase in the number of Lagrangian markers does not lead to any improvement in the representation of the immersed boundary. However, lower number of Lagrangian markers will contribute to under resolution of the immersed boundary as seen by the Eulerian grid on which the flow is resolved. If we follow this criterion for optimal number of Lagrangian markers for a non-uniform grid, the Lagrangian spacing will be dictated by the smallest Eulerian grid spacing surrounding the sphere. For a sphere lying close to the wall, this criterion, along with a uniform distribution of Lagrangian markers will result in an impractical number of Lagrangian markers. In the region close to the wall the spacing between the Lagrangian markers will be comparable to the fine Eulerian mesh and such high resolution is needed to resolve the near-wall flow features. As we move away from the wall, while the Eulerian grid spacing increases, a uniform distribution of Lagrangian markers will correspond to over-resolution, at the expense of significantly enhanced computational cost. To overcome this problem, an algorithm that generates a dynamic non-uniform distribution of Lagrangian markers on a sphere is proposed.

A related problem that this study addresses is the calculation of the Lagrangian volume weights assigned to the Lagrangian markers. These weights are used in the spreading step of IBM which can be expressed as,

$$
f(\boldsymbol{x})=\sum_{l=1}^{N_{L}} F\left(\boldsymbol{X}_{\boldsymbol{l}}\right) \delta\left(\boldsymbol{x}-\boldsymbol{X}_{\boldsymbol{l}}\right) \Delta V_{l},
$$

where $F\left(X_{l}\right)$ are the appropriate forcing required at the $N_{L}$ Lagrangian markers in order to satisfy the desired boundary conditions at the markers. The above expression is used to distribute these Lagrangian forcing to the surrounding Eulerian grid points to obtain $f(x)$, where $\delta\left(x-X_{l}\right)$ are the DDF used for 
spreading. Here $\Delta V_{l}$ are the volumetric weights assigned to each Lagrangian marker point. In the case of uniform distribution of Lagrangian markers, these volume weights are taken to be the same, as a constant. On a non-uniform grid Pinelli et al. (2010) suggests a mathematical method to calculate the weights by varying the thickness $\varepsilon$ of the interface shell as given by,

$$
f(\boldsymbol{x})=\sum_{l=1}^{N_{L}} F\left(\boldsymbol{X}_{\boldsymbol{l}}\right) \delta\left(\boldsymbol{x}-\boldsymbol{X}_{\boldsymbol{l}}\right) \varepsilon_{l} \Delta A_{l},
$$

where $\Delta A_{l}$ are the geometrical surface area associated with the Lagrangian markers. The main argument used by Pinelli et al. (2010) to calculate the thickness term $\varepsilon_{l}$ is the following. Starting with a Lagrangian function $F\left(X_{l}\right)$, it can be spread onto the Eulerian grid using equation (1.2). Then an Eulerian-to-Lagrangian interpolation follows using,

$$
F\left(\boldsymbol{X}_{\boldsymbol{l}}\right)=\sum_{\forall \boldsymbol{X}} f(\boldsymbol{x}) \delta\left(\boldsymbol{x}-\boldsymbol{X}_{\boldsymbol{l}}\right) \Delta x \Delta y \Delta z
$$

With the proper choice of $\varepsilon_{l}$, one should recover the same value of the initial value of $F\left(X_{l}\right)$ after going through the two steps.

Calculating the geometric surface area $\Delta A_{l}$ associated with a distribution of Lagrangian markers on a sphere is a challenging task. This study presents a novel numerical technique to calculate the surface areas for any distribution of Lagrangian markers, be it uniform or non-uniform. This new weight allocation reduces the numerical error even in the case of nearly even distribution of markers. It is being presented here in the context of IBM applications, but can be equally useful to numerical modeling in other fields, such as the minimum energy state of electrons on a unit sphere (Yudin 1993, Andreev 1996, Brown 2014), and arrangement of protein subunits on shells of spherical viruses (Klug et al. 1966).

The area weights of the Lagrangian markers are evaluated by requiring that the net force and moment on the sphere be computed as accurately as possible. The tractional force on the surface of the sphere is expressed as a multipole expansion of vector spherical harmonics. The appropriate values of the area weights are evaluated by requiring the discrete quadrature sum over all the Lagrangian markers with their respective area weights be equal to the analytically integrated net force and moment on the sphere. This equality is demanded for all three components of force and three components of moment for each vector spherical harmonic mode. We note that many of the resulting equations for the area weights are linearly dependent and a careful analysis will isolate the subset of independent equations. If we limit attention to only lower order terms of the multipole expansion, it is likely that the number of Lagrangian markers will exceed the number of independent equations. In which case, we propose to expand the area weights in scalar spherical harmonic modes and solve for only the lowest modes that equal the number of independent equations. As will be demonstrated this approach is robust to provide accurate values for the area weights that can be used in conjunction with IBM.

Finally it should be noted that the proposed non-uniform Lagrangian marker distribution and weights can be equally applied to other IBM methods like the more recent Particle-resolved Uncontaminated-fluid Reconcilable Immersed Boundary Method (PUReIBM) presented by Garg et al. (2011). The advantage of PUReIBM will be that it does not require forcing in the fluid phase. This method has been shown to conserve the transfer of turbulent kinetic energy between the solid and gas phase (Mehrabadi et al. 2015), and one can obtain the pressure and viscous forces over the surface of the sphere. Here for simplicity we demonstrate the proposed method in the context of direction forcing method of Uhlmann (2005).

The paper is structured as follows. Section 2 provides the geometry, governing equations and specifics of the numerical method used. Section 3 presents a brief description of the one-dimensional correction for the DDF in a non-uniform grid. Section 4 presents the algorithm to generate a non- 


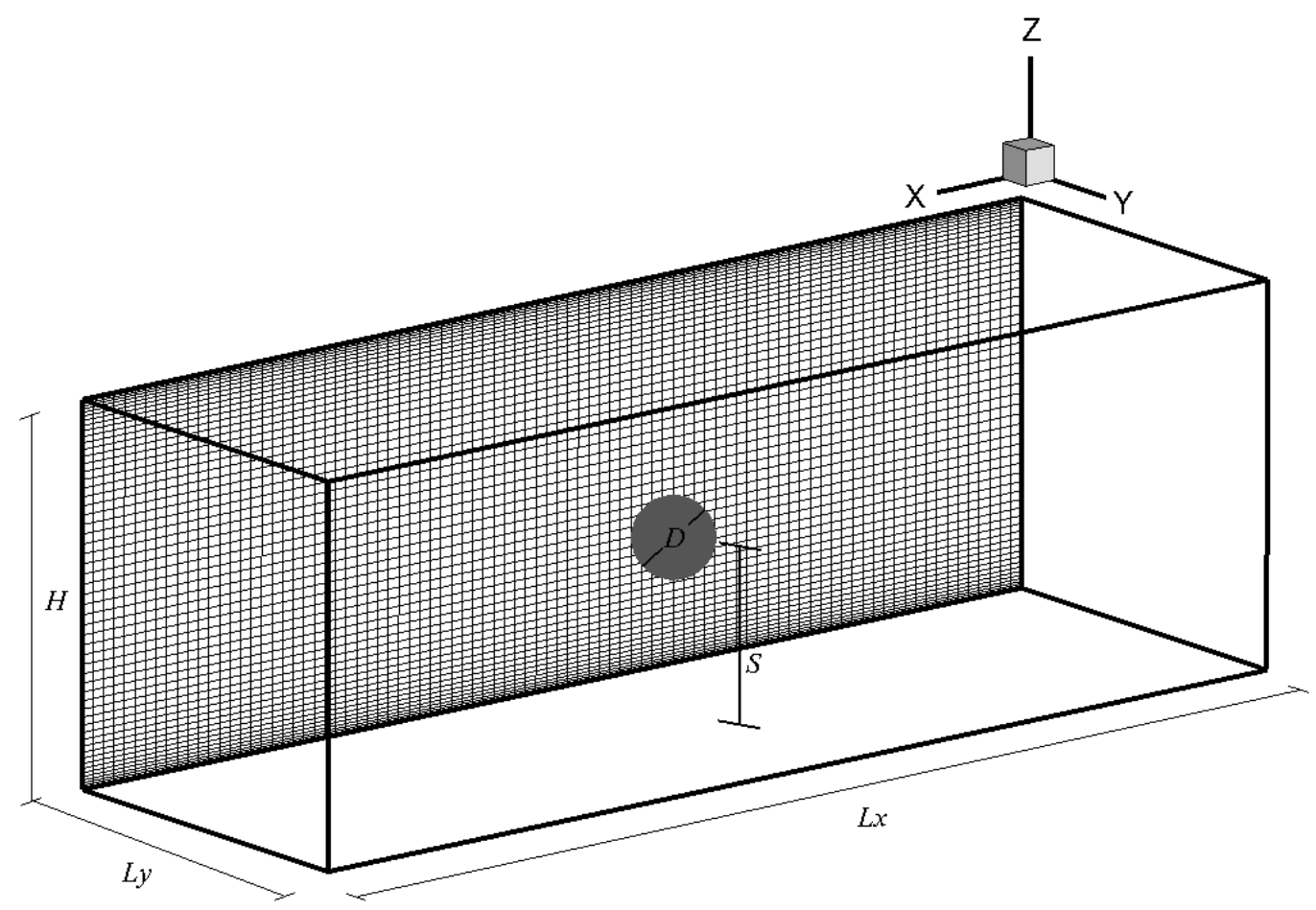

Figure 1. Schematic of the geometry.

uniform distribution of Lagrangian points on a sphere that matches the surrounding non-uniform Eulerian mesh. Section 5 describes in detail the numerical technique to calculate the Lagrangian volume weights that is used in the spreading function of the IBM. Section 6 demonstrates some results on the improvements obtained by applying the new techniques from Sections 4 and 5 . An overall validation of the code is given for computing the hydrodynamic forces and the motion of a sphere in a channel. Results are compared to analytical and numerical data from the literature. Also the potential computational savings and accuracy of the method presented is shown through simulations of a random cluster of spheres in a channel flow.

\section{Numerical Formulation}

Here we consider numerical simulation of a channel flow bounded only in the wall-normal direction. The governing equations are given by the incompressible Navier-Stokes equations,

$$
\begin{aligned}
& \nabla . \boldsymbol{u}=0 \\
& \frac{\partial \boldsymbol{u}}{\partial t}+\boldsymbol{u} . \nabla \boldsymbol{u}=\nabla p+\frac{1}{R e}\left(\nabla^{2} \boldsymbol{u}\right)+\boldsymbol{f} .
\end{aligned}
$$

$\boldsymbol{u}$ and $p$ are the non-dimensional velocity vector and pressure field respectively. The last term $\boldsymbol{f}$ in the momentum equation is the forcing term that is added to the fluid in order to account for additional immersed boundaries within the channel. Numerically, $f$ is sparse, with non-zero entries only in the vicinity of the Lagrangian markers that define the surface of the immersed boundary. The Reynolds number $R e$ is defined as $R e=U_{b} H / v$ where $U_{b}$ is the bulk velocity in the streamwise direction, $H$ is the channel height, and $v$ is the kinematic viscosity of the fluid. The time scale is $H / U_{b}$ and the pressure scale is $\rho U_{b}^{2}$, where $\rho$ is the density of the fluid. 
A schematic of the geometry is shown in Figure 1. The grid in the streamwise and spanwise direction, i.e. $x$ and $y$ directions, is uniform. In the case of spectral methodology, the mesh in the wallnormal direction ( $z$-direction) follows a Chebyshev Gauss-Lobatto quadrature given by,

$$
z_{k}=\cos \left[\frac{(k-1) \pi}{N_{z}-1}\right] \quad 1 \leq k \leq N_{z},
$$

where $N_{z}$ is the number of grid points in the z direction. The methodology to be presented here is equally applicable to other higher order discretizations, such as compact difference, where again it is advantageous to use a non-uniform grid in the $z$-direction by clustering the grid points closer to the channel walls. With compact and other higher-order finite difference methods, one is not restricted to quadrature based (Gauss-Lobatto) grid points.

The DNS uses a mixed third order Runge-Kutta and Crank-Nicolson scheme for the temporal discretization. At every time stage $m$, an intermediate velocity field is calculated by solving the Helmholtz equations before a pressure correction step is applied to render the velocity field divergence free. The Helmholtz equations are given by,

$$
\begin{aligned}
\nabla^{2} \boldsymbol{u}^{*(m)}-\frac{R e}{c d_{m}} \boldsymbol{u}^{*(m)}=-\frac{R e}{c d_{m}} & \left\{\boldsymbol{u}^{(m-1)}+c 1_{m} \Delta t[(\boldsymbol{u} . \nabla) \boldsymbol{u}]^{(m-1)}\right. \\
& \left.+c 1_{m} c 2_{m} \Delta t[(\boldsymbol{u} . \nabla) \boldsymbol{u}]^{(m-2)}+\frac{c d_{m}}{R e} \nabla^{2} \boldsymbol{u}^{(m-1)}+\Delta t \boldsymbol{f}^{(m)}\right\} .
\end{aligned}
$$

The coefficients $c d_{m}, c 1_{m}$, and $c 2_{m}$ are the coefficient of diffusion, coefficient of the first nonlinear term, and the coefficient of the second nonlinear term respectively. All the coefficients are characteristics of the time scheme used and are defined as (Canuto et al. 1988, Cortese \& Balachandar 1995),

$$
\begin{aligned}
& c d_{m}=\left\{\frac{\Delta t}{6}, \frac{5 \Delta t}{24}, \frac{\Delta t}{8}\right\} \\
& c 1_{m}=\left\{0,-\frac{5}{9},-\frac{153}{128}\right\} \\
& c 2_{m}=\left\{\frac{1}{3}, \frac{15}{16}, \frac{8}{15}\right\} .
\end{aligned}
$$

The pressure correction step is carried out through the following Poisson's equation,

$$
\nabla^{2} \phi^{(m)}=\frac{1}{2 c d_{m}} \nabla \cdot \mathbf{u}^{*(m)}
$$

where $\phi$ is the pseudo-pressure. The velocity and pressure fields are then updated accordingly,

$$
\begin{aligned}
& \boldsymbol{u}^{(m)}=\boldsymbol{u}^{*(m)}-2 c d_{m} \nabla \phi^{(m)} \\
& p^{(m)}=p^{(m-1)}+\phi^{(m)}-\frac{c d_{m}}{\operatorname{Re}} \nabla^{2} \phi^{(m)} .
\end{aligned}
$$

To obtain spectrally accurate derivatives, Fourier expansions are employed in the $x$ and $y$ direction. In the $z$-direction, Chebyshev expansion is employed.

As pointed out by Favier et al. (2014), the pressure correction step influences the imposed immersed boundary condition. Nevertheless, solutions based on this approach have been shown to be sufficiently accurate in a variety of problems (Van der Hoef et al. (2008), Apte et al. (2009), Feng et al. (2009), Dorai et al. (2015)). 


\subsection{Immersed Boundary Method}

To account for the immersed boundaries, a sphere in the present case, the forcing function $f$ in equation (2.4) is the only term that needs to be added to the above formulation. The way $f$ is calculated is the main source of difference between the various immersed boundary methods. For this study, the method introduced by Uhlmann (2005) is applied. The fluid-solid interface is marked by discrete Lagrangian markers. The total number of Lagrangian markers is denoted as $N_{L}$. At each Lagrangian marker, a specific force is calculated, which then is spread onto the Eulerian grid. This force ensures the desired velocity boundary condition at the immersed boundary. In the following brief description of the methodology, quantities on the Eulerian and Lagrangian grids are referred to by lowercase and uppercase letters respectively.

$$
\begin{aligned}
& \tilde{\boldsymbol{u}}=\boldsymbol{u}^{(m-1)}+c 1_{m} \Delta t[(\boldsymbol{u} . \nabla) \boldsymbol{u}]^{(m-1)}+c 1_{m} c 2_{m} \Delta t[(\boldsymbol{u} . \nabla) \boldsymbol{u}]^{(m-2)}+2 \frac{c d_{m}}{R e} \nabla^{2} \boldsymbol{u}^{(m-1)}-2 c d_{m} \nabla p^{(m-1)} \\
& \tilde{\boldsymbol{U}}\left(\underline{X}_{l}\right)=\sum_{\forall \underline{x}} \tilde{\tilde{u}}(\underline{x}) \delta\left(\underline{x}-\underline{X}_{l}\right) d v(\underline{x}) \\
& \boldsymbol{F}\left(\underline{X}_{l}\right)=\frac{\boldsymbol{U}\left(\underline{X}_{l}\right)^{(d)}-\tilde{\boldsymbol{U}}\left(\underline{X}_{l}\right)}{\Delta t} \\
& \boldsymbol{f}(\underline{x})=\sum_{l=1}^{N_{L}} \boldsymbol{F}\left(\underline{X}_{l}\right) \delta\left(\underline{x}-\underline{X}_{l}\right) \Delta V_{l}
\end{aligned}
$$

Eqn (2.9) is an explicit step which evaluates an approximate fluid velocity field on the Eulerian grid. In eqn (2.10), known as the interpolation step, the approximate Eulerian velocity is interpolated to the Lagrangian markers. Although the summation in eqn (2.10) is expressed to be over all the Eulerian grid points, the value $\widetilde{\boldsymbol{U}}$ at each Lagrangian point $X_{l}$ is influenced only by those Eulerian grid points in close proximity. This is ensured by the compact support of DDF, which extends over only a few Eulerian grid points around each Lagrangian marker (DDF will be discussed in Section 3). Here $d v(\underline{x})$ is the volume of the corresponding Eulerian grid. Eqn (2.11) calculates the Lagrangian forcing needed to render $\widetilde{U}\left(\underline{X}_{l}\right)$ equal to the desired velocity at the interface $\widetilde{U}\left(\underline{X}_{l}\right)^{(d)}$. Finally, the Lagrangian forcing is spread onto the Eulerian grid in eqn (2.12). The weight of each Lagrangian marker is denoted as $\Delta V_{l}$ which will be discussed in Section 5. Here again, due to the property of DDF, the additional force $f(\underline{x})$ to be applied at the Eulerian grid points is non-zero only in the neighborhood of the Lagrangian markers. Once the force $\boldsymbol{f}^{(m)}$ is determined (at each RK stage), the Helmoltz equations (2.4) are solved for each velocity component, followed by the pressure Poisson equation (2.6) and finally the correction step given by eqns (2.7) and (2.8).

\section{Distribution Function}

On a uniform grid the second order discrete delta function (DDF) involves three points in each direction. The expression of the 3D DDF is defined by Roma et al. (1999) as,

$$
\delta^{(3)}\left(\underline{x}-\underline{X}_{l}\right)=\delta_{h_{x}}^{(1)}\left(x-X_{l}\right) \delta_{h_{y}}^{(1)}\left(y-Y_{l}\right) \delta_{h_{z}}^{(1)}\left(z-Z_{l}\right)
$$

where 


$$
\begin{aligned}
& \delta_{h_{x}}^{(1)}\left(x-X_{l}\right)=\frac{1}{h_{x}} \psi\left(\frac{x-X_{l}}{h_{x}}\right), \\
& \delta_{h_{y}}^{(1)}\left(y-Y_{l}\right)=\frac{1}{h_{y}} \psi\left(\frac{y-Y_{l}}{h_{y}}\right), \\
& \delta_{h_{z}}^{(1)}\left(z-Z_{l}\right)=\frac{1}{h_{z}} \psi\left(\frac{z-Z_{l}}{h_{z}}\right) .
\end{aligned}
$$

The superscripts denote the dimension order and $h_{x}, h_{y}$ and $h_{z}$ are the dilation parameters, which define the characteristic distance between the grid points along each of the three directions. On a uniform grid, the dilation parameters are nothing but the grid spacing $\Delta x, \Delta y$, and $\Delta z$. The function $\psi$ is given by,

$$
\psi(r)= \begin{cases}\frac{1}{6}\left(5-3|r|-\sqrt{-3(1-|r|)^{2}+1}\right), & 0.5 \leq|r| \leq 1.5 \\ \frac{1}{3}\left(1+\sqrt{-3 r^{2}+1}\right), & |r| \leq 0.5 \\ 0, & \text { otherwise. }\end{cases}
$$

When a force is transferred from the Lagrangian marker to the Eulerian grid, the distribution function must ensure its conservation and the conservation of the first moment. Roma et al. (1999) defines these properties by,

$$
\begin{aligned}
& \sum_{\forall \underline{x}} \delta^{(3)}\left(\underline{x}-\underline{X}_{l}\right) \Delta v(\underline{x})=1 \\
& \sum_{\forall \underline{x}}\left(\underline{x}-\underline{X}_{l}\right) \delta^{(3)}\left(\underline{x}-\underline{X}_{l}\right) \Delta v(\underline{x})=0
\end{aligned}
$$

The DDF defined above satisfies these two conservation properties only if the Eulerian grid is uniformly spaced. In the case where the Eulerian mesh is non-uniform the DDF as defined above fails the conservation requirements. Also if a Lagrangian point is near a non-periodic boundary, such that the domain boundary falls within the limit $|r| \leq 0.5$ additional care is required in defining the DDF in order to satisfy the conservation constraints.

\subsection{Correction for DDF on a non-uniform grid}

Pinelli et al. (2010) presents an algorithm for IBMs in which non-uniform orthogonal or curvilinear grids can be utilized in conjunction with the DDF. In the present problem, the only direction that must be corrected is the wall-normal direction (i.e., $\delta_{h_{z}}^{1}\left(z-Z_{l}\right)$ must be re-defined). Following Pinelli et al. (2010) the modified delta function in the $z$-direction is calculated as follows.

$$
\delta_{h_{2}, \bmod }^{1}\left(z-Z_{l}\right)=\left[b_{0}+\left(z-Z_{l}\right) b_{1}+\left(z-Z_{l}\right)^{2} b_{1}\right] \delta_{h_{z}}^{1}\left(z-Z_{l}\right)
$$

The coefficients $b_{0}, b_{1}$, and $b_{2}$ are the solution of the following system of linear equations,

$$
\left(\begin{array}{lll}
m_{0} & m_{1} & m_{2} \\
m_{1} & m_{2} & m_{3} \\
m_{2} & m_{3} & m_{4}
\end{array}\right)\left(\begin{array}{l}
b_{0} \\
b_{1} \\
b_{2}
\end{array}\right)=\left(\begin{array}{l}
1 \\
0 \\
0
\end{array}\right)
$$

where 


$$
m_{i}=\sum_{k=1}^{N z}\left(z_{k}-Z_{l}\right)^{i} \delta_{h_{z}}^{1}\left(z_{k}-Z_{0}\right) \frac{\left(z_{k+1}-z_{k-1}\right)}{2} .
$$

The aforementioned dilation parameter $h_{z}$ needs to be adjusted accordingly and is defined as,

$$
h_{z}\left(Z_{l}\right)=\left(\frac{5}{6} h_{\max }+\frac{1}{6} h_{\min }+\varepsilon\right)
$$

where

$$
\begin{aligned}
& h_{\text {min }}\left(Z_{l}\right)=\min \left\{\left(z_{c-1}-z_{c-2}, z_{c}-z_{c-1}, z_{c+1}-z_{c}\right)\right\} \\
& h_{\text {max }}\left(Z_{l}\right)=\max \left\{\left(z_{c-1}-z_{c-2}, z_{c}-z_{c-1}, z_{c+1}-z_{c}\right)\right\}
\end{aligned}
$$

$z_{c}$ is the closest Eulerian point to the Lagrangian marker. Here $\varepsilon$ is a small fraction of the mesh width that is added to avoid having a singular $m$ matrix.

\subsection{Distribution Function for near wall Lagrangian Points}

Difficulty arises when the Lagrangian marker is close to the domain boundary (in the present case the channel walls) such that the nearest Eulerian point to the Lagrangian marker (in the definition of $z_{c}$ ) is the boundary node. In this case the non-uniform DDF defined above needs an additional (nonexistent) Eulerian grid point beyond the domain boundary. For these set of points, instead of using the 3point discrete delta function, the following 2-point distribution is used

$$
\begin{aligned}
& \delta\left(z_{i}-Z_{l}\right)=\frac{1}{2} \frac{z_{i+1}-Z_{l}}{\left(\left|z_{i+1}-Z_{l}\right|+\left|z_{i}-Z\right|\right)\left[z_{i+1}-z_{i}\right]} \\
& \delta\left(z_{i+1}-Z_{l}\right)=\frac{1}{2} \frac{Z_{l}-z_{i+1}}{\left(\left|z_{i+1}-Z_{l}\right|+\left|z_{i}-Z_{l}\right|\right)\left[z_{i+2}-z_{i}\right]} \\
& \delta\left(z_{k}-Z_{l}\right)=0
\end{aligned}
$$

where $z_{i}$ is the boundary node, $z_{i+1}$ is the second boundary node, $z_{i+2}$ is the third boundary node and $z_{k}$ represents all nodes beyond the second boundary node.

It should be noted that in a typical case, very few of the points would lie in the region where the 2-point distribution needs to be employed. Therefore, the effect on the accuracy of the total method is negligible.

\section{Lagrangian Points Distribution on a Sphere}

For a sphere immersed in a uniform Eulerian grid with a grid spacing $h$, the Lagrangian points are usually uniformly distributed such that each Lagrangian marker is associated with a surface area proportional to $h^{2}$. In other words, the spacing between the Lagrangian points on the surface of the immersed boundary must be of the order of grid spacing. For a spherical immersed boundary Uhlmann (2005) presents an equation to obtain the number of Lagrangian points $N_{L}$ in terms of the parameter $h$ and the sphere radius $r_{c}$,

$$
N_{L} \approx \frac{\pi}{3}\left(12 \frac{r_{c}^{2}}{h^{2}}+1\right)
$$

Uhlmann's study also shows that further increase in the number of Lagrangian markers does not lead to any enhancement of the solution. In the present case the Eulerian grid in the $z$-direction is non-uniform. An efficient method to generate the Lagrangian markers would be to allow their spacing to be of the 


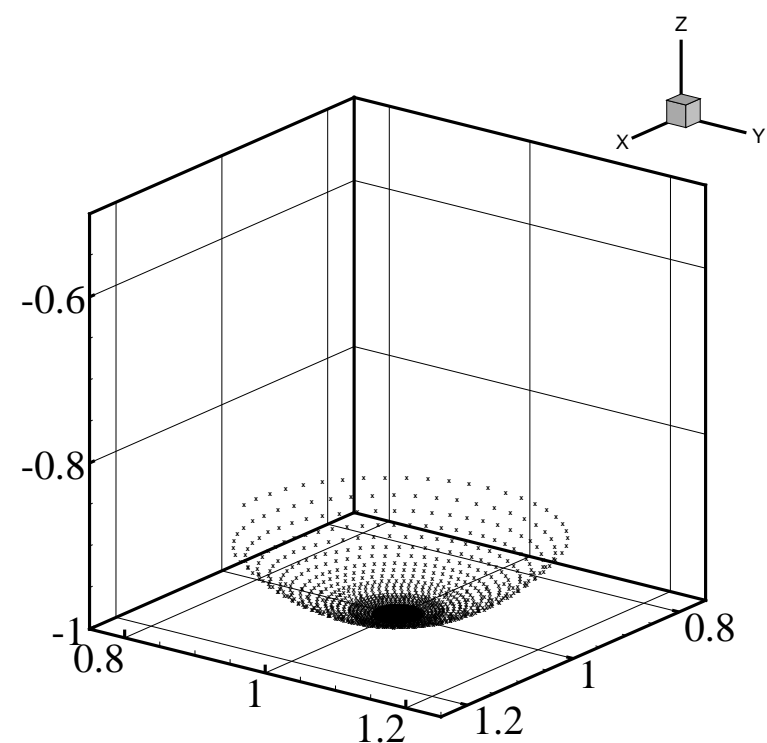

a)

\section{b)}

Figure 2. The spiral non-unifrom distribution of Lagrangian points on a sphere for a) 8 subsections and b) the entirety of the sphere.

order of the smallest local Eulerian grid spacing. In the context of present channel flow the grid spacing is uniform along the streamwise and spanwise directions and furthermore, the smallest Eulerian grid spacing is typically in the wall-normal direction. Thus, an efficient strategy will be to increase the spacing between the Lagrangian markers to be proportional to the local wall-normal grid spacing. This will require a fine spacing between the Lagrangian markers when they are close to the top or bottom walls, while the spacing between the Lagrangian markers can increase away from the channel walls. A simple algorithm to accomplish such a non-uniform distribution is given below.

Let the coordinate of the center of the sphere be denoted by $\left[X_{c}, Y_{c}, Z_{c}\right]$. The lowest point on the sphere has a $z$ coordinate of $Z_{\text {low }}=Z_{c^{-}} r_{c}$. The Eulerian grid spacing $\Delta z_{\text {low }}$ in which $Z_{\text {low }}$ lies is used as the spacing criterion $h$ in equation (4.1), i.e.,

$$
N_{l} \approx \frac{\pi}{3}\left(12 \frac{r_{c}^{2}}{\Delta z_{\text {low }}{ }^{2}}+1\right) .
$$

Using the spiral point distribution algorithm of Saff and Kuijlaars (1997), the Lagrangian markers can be generated starting from the lowest point on the sphere. In a spherical coordinate system, the polar coordinate $\theta$ (with respect to the sphere's center) of the markers can be obtained by

$$
\begin{aligned}
& h_{k}=-1+\frac{2(k-1)}{\left(N_{l}-1\right)} \quad 1 \leq k \leq N_{l} \\
& \theta_{k}=\arccos \left(h_{k}\right) .
\end{aligned}
$$

Then, the azimuthal coordinate is computed using the following equation,

$$
\left\{\begin{array}{l}
\phi_{k}=\left(\phi_{k-1}+\frac{3.809}{\sqrt{N_{l}}} \frac{1}{\sqrt{1-h_{k}^{2}}}\right)(\bmod 2 \pi) \\
\phi_{1}=\phi_{N_{l}}=0
\end{array}\right.
$$

The spherical coordinates are then transformed to the Cartesian system, 


$$
\begin{aligned}
& X_{k}=X_{c}+r_{c} \sin \left(\theta_{k}\right) \cos \left(\phi_{k}\right) \\
& Y_{k}=Y_{c}+r_{c} \sin \left(\theta_{k}\right) \sin \left(\phi_{k}\right) \\
& Z_{k}=Z_{c}+r_{c} \cos \left(\theta_{k}\right) .
\end{aligned}
$$

The loop over $k$ if continued from 1 to $N_{l}$ will generate the Lagrangian markers for the whole sphere. This distribution of Lagrangian markers would be approximately uniform, but will result in numerous Lagrangian markers and computationally wasteful over-resolution. To avoid that, the loop is stopped once $Z_{k}$ crosses the $z$-location of the Eulerian grid point that defines the local grid spacing $\Delta z_{\text {low }}$. At this point, $\Delta z_{\text {low }}$ in equation (4.2) is replaced by the next Eulerian grid spacing $\Delta z_{\text {low }+1}$ to obtain a new $N_{l}$. which is then used to generate the next section of Lagrangian markers over the sphere between the two Eulerian grid points defining $\Delta z_{l o w+1}$. These sections are denoted as "subsections" for future reference. The subsections are generated in sequential order until the surface of the sphere is completely described by the Lagrangian markers. Figure 2 shows a sphere of radius 0.5 with the center located at $[1,1,-0.5]$ in a channel that extends from -1 to 1 in the wall-normal direction. Figure 2a shows the semi-constructed sphere after completing 8 subsections. Figure $3 \mathrm{~b}$ shows the complete distribution of Lagrangian markers. It can be clearly seen how the spacing becomes much coarser as we move away from the bottom boundary commensurate with the coarsening of the local wall-normal Eulerian grid.

In this study, the spiral method has been used within each subsection for demonstration. The same logic can be applied using different "uniform" distribution of points method within each subsection.

\section{Lagrangian Volume Weights}

Upon distributing the Lagrangian markers on a sphere, a weight assigned with each point is required to perform the spreading step defined in equation (2.12). Historically, for a uniform distribution of Lagrangian markers immersed in a uniform Eulerian grid, $\Delta V_{l}$ has been taken as a constant equal to $\Delta x^{3}$. In the case of a non-uniform distribution of Lagrangian markers, the volume weights need to be varied according to the manner in which the markers are spatially distributed on the immersed interface.

A perfectly uniform distribution of markers around a sphere can be achieved only in the case when the number of Lagrangian markers is small. Uniform distribution of markers on a sphere for $N_{L}>12$ remains an unsolved problem in the mathematics and physics community, known as the Thompson problem (1904). Many approximate methods can be found in the literature to distribute an arbitrary number of points on a sphere, but none are exact. Lagrangian marker distribution algorithms, such as the one suggested by Saff and Kuijlaars (1997), is only approximate. Therefore the assumption of uniform weight will necessarily involve error, which may be small, but non-zero.

In this section, a method is presented to assign weight for each marker on a sphere for any distribution, be it uniform or random. This new weight allocation can compensate for the numerical error associated with inexact uniform distribution of Lagrangian markers. It is being presented here in the context of IBM applications, but can be equally useful in other fields, where a uniform distribution of Lagrangian markers on a sphere are required. Determining the appropriate values of the weights is achieved by requiring the discrete quadrature to exactly recover the analytically integrated force on the immersed spherical surface for known force distributions. These analytically integrated force distributions will be discussed below. 


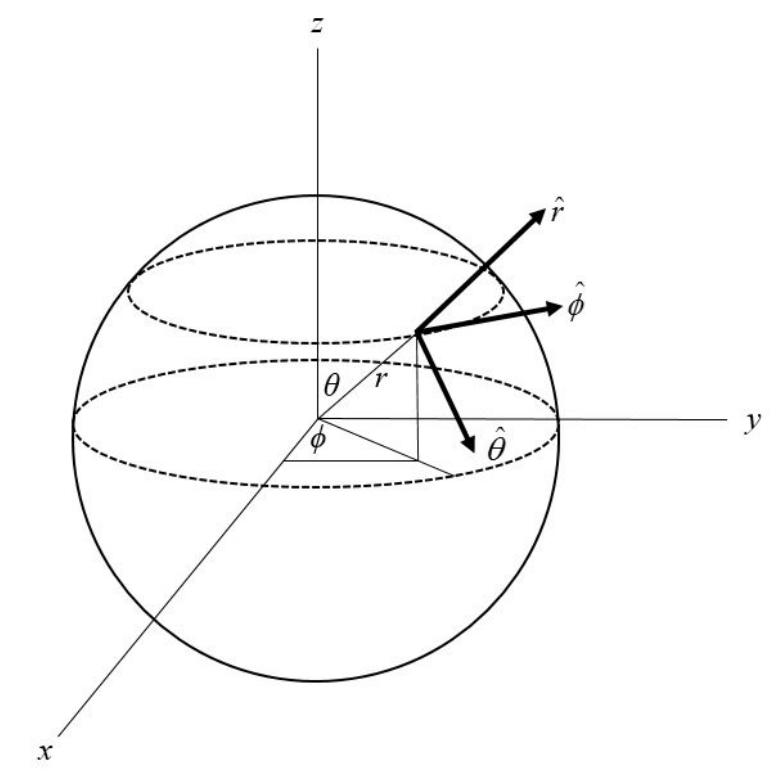

Figure 3. Schematic of the polar coordinates and vectors.

\subsection{Multipole Expansion of Surface Traction}

The tractional force on the spherical interface is expressed in terms of vector spherical harmonics (VHS). Following Barrera et al. (1985), we define three VSH in spherical coordinate $(r, \theta, \quad)$ in terms of a scalar spherical harmonic function as (refer to Figure 3)

$$
\begin{aligned}
& \mathbf{Y}_{i j}=Y_{i j} \hat{r} \\
& \boldsymbol{\Psi}_{i j}=r \nabla Y_{i j} \\
& \boldsymbol{\Phi}_{i j}=r \hat{r} \times \nabla Y_{i j}
\end{aligned}
$$

where $\hat{r}$ is the unit vector along the radial direction and the scalar spherical harmonic function is given by

$$
Y_{i j}=F_{i j, c} P_{i}^{j}(\cos \theta) \cos (j \phi)+F_{i j, s} P_{i}^{j}(\cos \theta) \sin (j \phi) .
$$

Here $j$ indicates the wave number in the azimuthal direction, while $i$ is the mode number in the tangential direction ( $i$ corresponds to the number of zero crossings of the Legendre polynomials). Thus $F_{i j, c}$ and $F_{i j, s}$ determine the amplitudes of the scalar harmonic modes of wave number $(i, j)$ in the tangential and azimuthal directions. Note that the harmonics $\mathbf{Y}_{i j}$ are oriented along the radial direction, while the other two vector harmonics are oriented tangential to the surface of the sphere. It should be noted that both $\boldsymbol{\Psi}_{i j}$ and $\boldsymbol{\Phi}_{i j}$, although perpendicular to each other, are not purely oriented in either $\theta$ or direction. From the definition of $Y_{i j}$ it can be seen that all three vector spherical harmonics are functions of $\theta$ and coordinates. A surface vector field is then defined as the following multipole expansion

$$
\mathbf{E}=\sum_{i=0}^{I} \sum_{j=0}^{i} E_{i j, r} \mathbf{Y}_{i j}+E_{i j, \psi} \mathbf{\Psi}_{i j}+E_{i j, \phi} \mathbf{\Phi}_{i j},
$$

where the first term on the right is the radial component, while the other two together account for the tangential components. When the vector spherical harmonics are substituted into the multipole 
expansion, the constants in equations (5.2) and (5.3) can be merged to define a unique set of new coefficients

$$
\begin{array}{lll}
F_{i j, r c}=F_{i j, c} E_{i j, r} & F_{i j, \Psi c}=F_{i j, c} E_{i j, \Psi} & F_{i j, \Phi c}=F_{i j, c} E_{i j, \Phi} \\
F_{i j, r s}=F_{i j, s} E_{i j, r} & F_{i j, \Psi s}=F_{i j, s} E_{i j, \Psi} & F_{i j, \Phi s}=F_{i j, s} E_{i j, \Phi} .
\end{array}
$$

For any chosen value of $I$ the above multipole expansion offers a systematic way of representing vector valued functions on the surface of the sphere. With increasing value of $I$ more complex variations of traction vector on the surface of the sphere can be represented. For example, for $I=1$, equation (5.3) reduces to,

$$
\begin{aligned}
\mathbf{E}=( & \left.F_{00, r c}+F_{10, r c} \cos \theta-\sin \theta\left(F_{11, r c} \cos \phi+F_{11, r s} \sin \phi\right)\right) \hat{r} \\
& +\left(-F_{10, \Psi_{c}} \sin \theta-F_{11, \Psi_{c}} \cos \theta \cos \phi-F_{11, \Psi_{s}} \cos \theta \sin \phi+F_{11, \Phi c} \sin \phi-F_{11, \Phi s} \cos \phi\right) \hat{\theta} \\
& +\left(F_{10, \Phi c} \sin \theta+F_{11, \Psi_{c}} \sin \phi-F_{11, \Psi_{s}} \cos \phi+F_{11, \Phi c} \cos \theta \cos \phi+F_{11, \Phi s} \cos \theta \sin \phi\right) \hat{\phi} .
\end{aligned}
$$

$\mathbf{E}$ embodies the sum of several spherical harmonic modes of force distribution on a sphere. For example, the $(i=1, j=0)$ mode contributes only to a uniform radial force, whose magnitude is given by the coefficient $F_{00, r c}$. In case of $(i=1, j=0)$ only the cosine mode contributes to all three components of tractional force (i.e., $F_{10, s}=0$ ).

Upon integrating the tractional force distribution $\mathbf{E}$ over the surface of the sphere one can analytically obtain the net resultant force and moment on the sphere as

$$
\mathbf{F}=\oiint_{S} \mathbf{E} d \theta d \phi \quad \text { and } \quad \mathbf{M}=\oiint_{S} r(\hat{r} \times \mathbf{E}) d \theta d \phi
$$

Substituting equation (5.5) into equation (5.6), the three Cartesian components of force and moment are obtained as

$$
\begin{aligned}
& \mathbf{F}=\left\{\begin{array}{l}
{\left[-\frac{4}{3} F_{11, r c} \pi r^{2} \hat{i}-\frac{4}{3} F_{11, r s} \pi r^{2} \hat{j}+\frac{4}{3} F_{10, r c} \pi r^{2} \hat{k}\right]+} \\
{\left[-\frac{2}{3} F_{11, \Psi c} \pi r^{2} \hat{i}-\frac{2}{3} F_{11, \Psi s} \pi r^{2} \hat{j}+\frac{8}{3} F_{10, \Psi c} \pi r^{2} \hat{k}\right]+} \\
{\left[-2 F_{11, \Psi c} \pi r^{2} \hat{i}-2 F_{11, \Psi s} \pi r^{2} \hat{j}\right]}
\end{array}\right. \\
& \mathbf{M}=\left\{\begin{array}{l}
{[0]+} \\
{\left[-2 F_{11, \Phi c} \pi r^{3} \hat{i}-2 F_{11, \Phi s} \pi r^{3} \hat{j}\right]+} \\
{\left[\begin{array}{l}
2 \\
\left.-\frac{2}{3} F_{11, \Phi c} \pi r^{3} \hat{i}-\frac{2}{3} F_{11, \Phi s} \pi r^{3} \hat{j}+\frac{8}{3} F_{10, \Phi c} \pi r^{3} \hat{k}\right]
\end{array}\right.}
\end{array}\right.
\end{aligned}
$$

Here $\hat{i}, \hat{j}$ and $\hat{k}$ are the unit vectors along $x, y$ and $z$ directions. In the above expression for the integrated force, the three components within the first parenthesis are from the integration of the radial component of the multipole expansion given in (5.5), the terms in the second and third parenthesis arise from the integration of the tangential and azimuthal components. Similarly, in the expression of the moment, the terms within the three parenthesis arise from the radial, tangential and azimuthal components of the multipole expansion. The derivation of equations (5.7) and (5.8) is shown in Appendix A. The above process can be followed systematically to obtain higher-order terms of the multipole expansion. It can be shown that all the resultant forces and moments of modes greater than $(i$ 
$=1, j=1)$ are identically zero. These modes represent tractional surface force distribution of higher wavenumber that might be encountered in turbulent flows around a sphere but have no contribution to the net hydrodynamic force or moment on the particle.

\subsection{Lagrangian Weights Through Numerical Quadrature}

The integrated force and moment expressions obtained in equations (5.7) and (5.8) can be used to obtain the Lagrangian weights. Once the Lagrangian marker positions on the surface of the sphere are determined (for example using the method outlined in §4), for any chosen multipole expansion (i.e., for chosen values of the coefficients $F_{00, r c}, F_{10, r c}$, etc) the tractional force at each Lagrangian marker can be evaluated from (5.5) with the $r, \theta$, location of the marker with respect to the center of the sphere. Once the force at each Lagrangian marker is known, the net force and moment on the sphere can be obtained by numerically integrating over the entire surface of the sphere as expressed by the following summations:

$$
\mathbf{F}_{\text {num }}=\sum_{l=1}^{N_{L}} \mathbf{E}\left(X_{l}\right) A_{l} \quad \text { and } \quad \mathbf{M}_{\text {num }}=\sum_{l=1}^{N_{L}}\left[X_{l} \times \mathbf{E}\left(X_{l}\right)\right] A_{l}
$$

where $A_{l}$ are the area weights associated with the Lagrangian markers. The central idea is to evaluate the optimal value of these weights by requiring that the above numerical approximations be equal to the exact values, which can be obtained from (5.7) and (5.8).

Since the number of Lagrangian markers, and correspondingly the number of weights, $N_{L}$ is typically large we need several equations of equality between the exact and the numerical approximations. This can be accomplished by different modal approximations of the multipole force distribution. One modal approximation will be to set $F_{00, r c}=1$ and all other coefficients equal to zero in (5.5). From equations (5.7) and (5.8) we see that this mode corresponds to a purely radial force distribution and thus contributes to zero integrated net force and zero integrated net moment. Another modal approximation will be to set $F_{10, \Psi_{c}}=1$ and all other coefficients in (5.5) zero, which results in the following tractional force distribution

$$
\mathbf{E}=-\sin \theta \hat{\theta} .
$$

According to equations (5.7) and (5.8), upon integration over the surface of the sphere the resulting net force has only $z$-component of magnitude $8 \pi r^{2} / 3$ and has zero moment. In order to calculate the corresponding numerical values of the net force and moment, the surface force distribution given in (5.10) is evaluated at every Lagrangian marker whose polar coordinates are known. The weighted sum over all the Lagrangian markers should recover the analytical solutions of the net integrated force along the $x$-direction, which can be expressed as

$$
F_{x}=\sum_{l=1}^{N_{L}}\left(-\sin \theta_{l}\right)\left(\cos \theta_{l} \cos \phi_{l}\right) A_{l}=0 .
$$

Likewise, equating the remaining components of the numerically integrated force components and moments to their analytical counterparts we obtain

$$
\begin{aligned}
& F_{y}=\sum_{l=1}^{N_{L}}\left(-\sin \theta_{l}\right)\left(\cos \theta_{l} \sin \phi_{l}\right) A_{l}=0 \\
& F_{z}=\sum_{l=1}^{N_{L}}\left(-\sin \theta_{l}\right)\left(-\sin \theta_{l}\right) A_{l}=\frac{8}{3} \pi r^{2} \\
& M_{x}=\sum_{l=1}^{N_{L}}\left(-\sin \theta_{l}\right)\left(-\sin \theta_{l}\right) A_{l} \Delta y_{l}-\left(-\sin \theta_{l}\right)\left(\cos \theta_{l} \sin \phi_{l}\right) A_{l} \Delta z_{l}=0
\end{aligned}
$$




$$
\begin{aligned}
& M_{y}=\sum_{l=1}^{N_{L}}\left(-\sin \theta_{l}\right)\left(\cos \theta_{l} \cos \phi_{l}\right) A_{l} \Delta z_{l}-\left(-\sin \theta_{l}\right)\left(-\sin \theta_{l}\right) A_{l} \Delta x_{l}=0 \\
& M_{z}=\sum_{l=1}^{N_{L}}\left(-\sin \theta_{l}\right)\left(\cos \theta_{l} \sin \phi_{l}\right) A_{l} \Delta x_{l}-\left(-\sin \theta_{l}\right)\left(\cos \theta_{l} \cos \phi_{l}\right) A_{l} \Delta y_{l}=0,
\end{aligned}
$$

where $\Delta x_{l}, \Delta y_{l}$, and $\Delta z_{l}$ are the Cartesian coordinates of the Lagrangian points with respect to the center of the sphere, defined as,

$$
\begin{aligned}
& \Delta x=r \sin \theta \cos \phi \\
& \Delta y=r \sin \theta \sin \phi \\
& \Delta z=r \cos \theta .
\end{aligned}
$$

The above eqns (5.11)-(5.16) can be considered as six relations for evaluating the unknown weights $A_{l}$. Thus, for each modal approximation, where one of the spherical harmonic coefficients is set equal to one, while all other coefficients are set to zero, we obtain a set of six relations for the Lagrangian weights.

If we limit our attention to $I=1$, then there are ten coefficients in equation (5.5). For each of these spherical harmonic modes we obtain six linear equations of the kind given in (5.11) to (5.16). These sixty equations can be written in a matrix form as

$$
\boldsymbol{M} \vec{a}=\vec{b},
$$

where vector $\overrightarrow{\boldsymbol{a}}$ contains the unknown weights $A_{l}$ and vector $\overrightarrow{\boldsymbol{b}}$ holds the analytical integrated forces and moments presented in equations (5.7) and (5.8). Matrix $\boldsymbol{M}$ has several linearly dependent rows which can be sorted out (see Appendix A.2 for details). This results in nine linearly independent equations which are listed below:

$$
\begin{aligned}
& \sum_{l=1}^{N_{L}}\left(\sin \theta_{l} \cos \phi_{l}\right) A_{l}=0 \\
& \sum_{l=1}^{N_{L}}\left(\sin \theta_{l} \sin \phi_{l}\right) A_{l}=0 \\
& \sum_{l=1}^{N_{L}}\left(\cos \theta_{l}\right) A_{l}=0 \\
& \sum_{l=1}^{N_{L}}\left(-\sin \theta_{l} \cos \phi_{l}\right)\left(\sin \theta_{l} \cos \phi_{l}\right) A_{l}=-\frac{4}{3} \pi r^{2} \\
& \sum_{l=1}^{N_{L}}\left(-\sin \theta_{l} \cos \phi_{l}\right)\left(\cos \theta_{l}\right) A_{l}=0 \\
& \sum_{l=1}^{N_{L}}\left(-\sin \theta_{l} \sin \phi_{l}\right)\left(\sin \theta_{l} \sin \phi_{l}\right) A_{l}=-\frac{4}{3} \pi r^{2} \\
& \sum_{l=1}^{N_{L}}\left(-\cos \theta_{l} \cos \phi_{l}\right)\left(\cos \theta_{l} \cos \phi_{l}\right) A_{l}+\left(\sin \phi_{l}\right)\left(-\sin \phi_{l}\right) A_{l}=-\frac{8}{3} \pi r^{2} \\
& \sum_{l=1}^{N_{L}}\left(-\cos \theta_{l} \sin \phi_{l}\right)\left(\cos \theta_{l} \cos \phi_{l}\right) A_{l}+\left(-\cos \phi_{l}\right)\left(-\sin \phi_{l}\right) A_{l}=0 \\
& \sum_{l=1}^{N_{L}}\left(-\cos \theta_{l} \sin \phi_{l}\right)\left(-\sin \theta_{l}\right) A_{l}=0 .
\end{aligned}
$$


In summary, the multipole expansion (5.3) for $I=1$ yields 10 different force distributions that are given in an expanded form in (5.5). For each surface force distribution we can compute the three components of surface integrated force and three components of surface integrated moments, both analytically (see eqns. 5.7 and 5.8), and as a quadrature sum over the Lagrangian markers. By requiring that the quadrature sums be equal to the analytical forces and moments, we thus obtain 60 linear relations (for $I=1$ ) for the to-be-determined Lagrangian area weights. Careful analysis shows that only nine of these 60 equations are linearly independent, resulting in the nine equations (5.19) to (5.27) for the $N_{l}$ area weights. It can be readily shown that for an arbitrary $I$, the number of force distributions (corresponding to 5.5) is given by

$$
C=1+3 I+6 \sum_{i=0}^{I} i
$$

For example, $I=2$ will result in 25 surface force distributions and by requiring that the quadrature sums be equal to the analytical forces and moments for each of these distributions, we obtain a total of $6 \times 25=150$ linear equations for the area weights. Again only a small fraction of these equations are linearly independent. Thus, by increasing $I$, we can in principle obtain as many linearly independent equations as needed for the unknown area weights. Note that these additional equations for $I=2$ and higher (i.e., equations beyond the nine given in 5.19 to 5.27 ) will require that the quadrature sum accurately capture the zero net force and zero net moment of the higher order surface force distributions.

\subsection{Solution of the Under-determined System}

In section 5.1 we noted that $I=1$ captures all the non-zero contributions to integrated forces and moments. Therefore, here we limit attention to the nine linearly independent equation that arise for $I=1$. However, since $N_{l}$ is typically larger than nine, we have an under-determined system.

To reduce the number of unknowns, the area weights are also expanded in terms of spherical harmonics as given by,

$$
A_{l}=\sum_{p=0}^{P} \sum_{q=0}^{p} \Delta A_{c, p, q} P_{p}^{q}\left(\cos \theta_{l}\right) \cos \left(q \phi_{l}\right)+\Delta A_{s, p, q} P_{p}^{q}\left(\cos \theta_{l}\right) \sin \left(q \phi_{l}\right)
$$

The total number of weight coefficients $\Delta A_{p, 0}, \Delta A_{c, p, q}$ and $\Delta A_{s, p, q}$ is $(P+1)^{2}$. Note that the second term vanishes when $q=0$. If we set $P$ to be 2 , we can match the number of unknown coefficients to the nine independent equations available for $I=1$. After solving for the nine unknowns coefficients, all the area weights can be found from equation (5.29). This approach has the advantage of ensuring that the Lagrangian weights have a smooth distribution around the surface of the sphere. However, the weights obtained this way will not capture the net force and moment due to higher-order variations of surface tractional forces. If needed, the above approach can be extended to include higher-order modes by increasing the value of $I$ in equation (5.3) and the value of $P$ in equation (5.29).

To obtain the volumetric weights used in the spreading equation (2.12), the area weights are multiplied by a constant thickness taken to be the square root of the mean values of $A_{l}$ in each subsection

$$
\Delta V_{i}=A_{i} \sqrt{\frac{4 \pi r^{2}}{N_{l}}}
$$

where $N_{l}$ is a parameter designated to each subsection calculated from equation (4.2). Uhlmann (2005) has defined the thickness to be equal to one grid spacing in a uniform Eulerian mesh. The thickness given in equation (5.30) applies this definition but on a local level to account for the mesh nonuniformity. One can also use the thickness calculation method for non-uniform grids from Pinelli et al. (2010). But care is needed, especially near the wall, where the stretching of the grid is severe. 
Table 1. Number of Lagrangian points at various heights $S$ for a sphere of radius $R=1 / 16$ ( $H=1$ and $\left.N_{z}=97\right)$.

\begin{tabular}{lll}
\hline Height $S$ & Uniform Distribution & Non-uniform Distribution \\
\hline \hline $8 R$ (center of the channel) & 186 & 184 \\
$6 R$ & 213 & 196 \\
$4 R$ & 299 & 250 \\
$3 R$ & 407 & 310 \\
$2 R$ & 778 & 453 \\
$1.5 R$ & 1616 & 639 \\
$1.25 R$ & 3106 & 851 \\
$1.01 R$ & 76159 & 1888 \\
$R$ (tangent to the wall) & 684941 & 3742 \\
\hline
\end{tabular}

It should be noted that the application of this method would only require solving for the 9 area weights coefficients $\Delta A$ using the 9 equations given in (5.19)-(5.27) after substituting the Lagrangian areas $A_{l}$ by the spherical harmonics expansion given in equation (5.29). The 9 linearly independent equations are valid for all Lagrangian markers distribution and do not need to be re-derived.

\section{Results}

\subsection{Number of Lagrangian Markers}

One of the goals is to minimize the number of Lagrangian markers on a sphere immersed in a nonuniform surrounding Eulerian mesh, without compromising accuracy. Other than the diameter of the sphere, the two main factors that affect the number of Lagrangian markers are the distance $S$ between the sphere and the wall (refer to Figure 1), and the number of Eulerian grid points in the $z$-direction, $N_{z}$.

First we test the efficiency of the proposed non-uniform distribution of the Lagrangian markers in a channel of unit non-dimensional height $(H=1)$ with a modest number of grid points along the wall normal direction $\left(N_{z}=97\right)$. The sphere's non-dimensional radius is chosen to be $R=1 / 16$. At several heights $S$, the Lagrangian markers are generated in two different ways. Once using the method outlined in $\S 4$, leading to a non-uniform distribution with spacing between the Lagrangian markers matching the background mesh. This will be compared against a uniform distribution of Lagrangian markers generated based on the smallest Eulerian grid spacing $\Delta z$ surrounding the sphere. In this second approach we will use eqn (4.1) with $h$ to be the smallest wall-normal grid spacing seen by the particle to calculate $N_{L}$ and spread the Lagrangian markers uniformly. The results obtained are reported in Table 1.

For a sphere lying at the center of the channel, both the uniform and non-uniform distributions result in nearly the same number of Lagrangian markers. The Gauss-Lobatto distribution of Eulerian grid points given in (2.3) is nearly uniform towards center of the channel. Since the non-uniform distribution matches the background mesh, the non-uniform Lagrangian markers in this case closely match those of the uniform distribution. In contrast, the closer the sphere is located to the wall, the greater the difference is between the two methods. In the limit where the sphere is in contact with the wall, the number of Lagrangian markers is reduced by 183 times with the use of the present non-uniform distribution of Lagrangian markers. In fact, without the non-uniform distribution, the number of uniformly distributed Lagrangian markers on a single sphere located on the wall would be $O\left(10^{6}\right)$, 

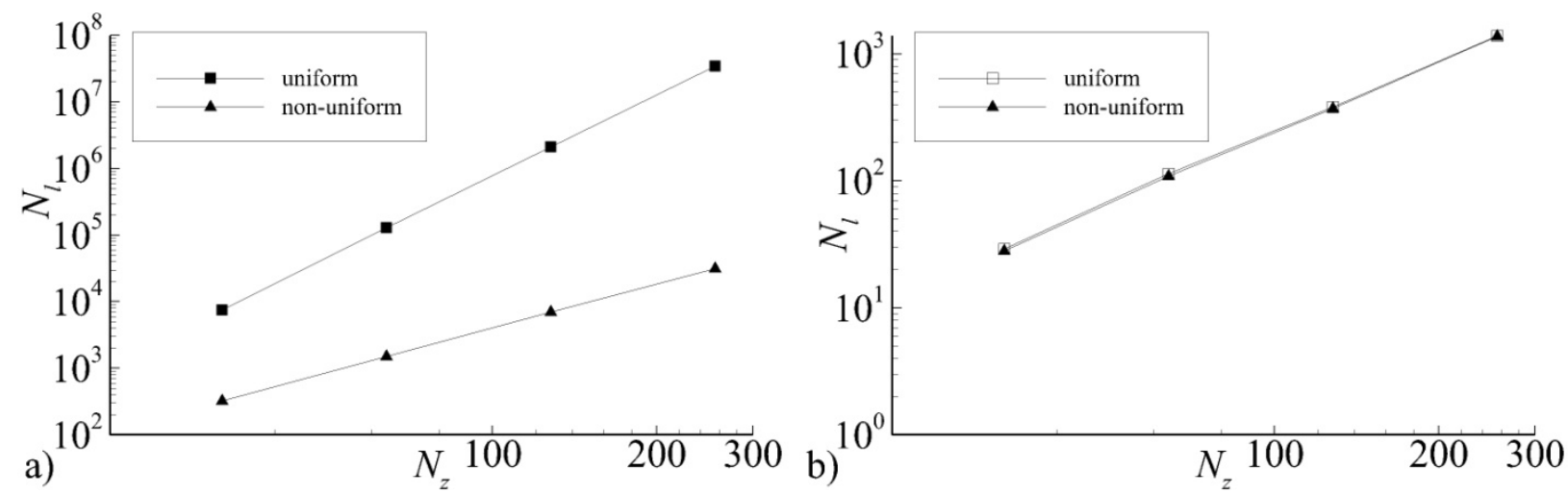

Figure 4. Number of Lagrangian points versus number of wall-normal Eulerian points for a sphere at a) the wall and $b)$ the center of the channel.

which is comparable to the total number Eulerian grid points used for the entire channel. Simply using less Lagrangian markers, while maintaining a uniform distribution, will lead to poor resolution of the immersed surface close to the wall, where the local Eulerian grid resolution will be an order of magnitude more than the Lagrangian resolution. Such under resolution of the Lagrangian interface will lead to "leaks" through the immersed boundary, as the desired velocity boundary condition on the immersed interface cannot be maintained on the finer surrounding Eulerian mesh. Thus a non-uniform distribution of Lagrangian markers is essential to limiting the computational cost, while maintaining the desired level of computational accuracy.

The number of Lagrangian points per sphere is also influenced by the variation of the number of Eulerian grid points in the $z$-direction. For this test, $S$ is kept constant and several values of $N_{z}$ are investigated. Figure 4a shows a plot of $N_{L}$ versus $N_{z}$ on a $\log \log$ scale for $S=R$ (i.e., for a sphere located on the wall). A significant reduction in the slope of the curve can be seen for the proposed nonuniform distribution of Lagrangian markers. For the parameters used, we obtain the following approximate scaling

$$
\begin{array}{ll}
N_{L} \sim N_{z}^{4.05} & \text { for uniform spacing. } \\
N_{L} \sim N_{z}^{2.2} & \text { for non-uniform spacing. }
\end{array}
$$

Figure $4 \mathrm{~b}$ shows the same plots but for $S=8 R$ (i.e., sphere located in the center of the channel). As expected, the results are nearly identical for both the uniform and non-uniform distribution algorithms. The correlation for the number of Lagrangian markers is now given by,

$$
N_{L} \sim O\left(N_{z}^{1.86}\right) \text {. }
$$

In spectral methods the number of floating point operations when solving the Helmholtz and Poisson's equations for the calculation of the velocity and pressure fields is of the order of $\max \left\{N_{x}, N_{y}, N_{z}\right\} \times N_{x} \times N_{y} \times N_{z}$. Implementation of IBM requires additional steps of interpolation from Eulerian-to-Lagrangian markers and spreading of Lagrangian force back onto the Eulerian mesh. These additional computations scale with the total number of Lagrangian points. In a simulation involving $N_{p}$ number of spheres, with each on average resolved by $N_{L}$ number of Lagrangian markers, the total number of markers will scale as $N_{p} \times N_{L}$ (Uhlmann 2005). In the majority of previous particle-resolved multiphase flow studies (Gilmanov \& Sotiropoulos 2005, Lee \& Balchandar 2010, Kidanemariam \& Uhlmann 2014, Uhlmann \& Doychev 2014), the Eulerian grid was chosen to be uniform with the same grid spacing along all three directions (i.e., $\Delta x=\Delta y=\Delta z$ ). Even with a large number of spheres, the total number of Lagrangian markers was comparable to the Eulerian grid and thus the implementation of IBM does not severely slow down the underlying fluid flow calculation. With a non-uniform grid resolution 
Table 2. Comparison of the analytical and numerical integration for CASE1 and CASE2 for non-zero values of resultant forces and moments.

\begin{tabular}{|c|c|c|c|c|c|c|}
\hline $\begin{array}{l}\text { Force } \\
\text { Profile }\end{array}$ & $\begin{array}{l}\text { Force/Moment } \\
\text { Direction }\end{array}$ & Analytical & $C A S E 1$ & $\%$ Error & CASE2 & $\%$ Error \\
\hline$F_{10, r c}$ & $F_{z}$ & 1.0471976 & 1.0488651 & $0.159 \%$ & 1.0471976 & $2.12 \times 10^{-14} \%$ \\
\hline$F_{11, r c}$ & $F_{x}$ & -1.0471976 & -1.0451141 & $0.199 \%$ & -1.0471976 & $2.12 \times 10^{-14_{0}} \%$ \\
\hline$F_{11, r s}$ & $F_{y}$ & -1.0471976 & -1.0451142 & $0.199 \%$ & -1.0471976 & $0 \%$ \\
\hline$F_{10, \psi c}$ & $F_{z}$ & 2.0943951 & 2.0902283 & $0.199 \%$ & 2.0943951 & $2.12 \times 10^{-14} \%$ \\
\hline$F_{10, \phi c}$ & $M_{z}$ & 1.0471976 & 1.0451142 & $0.199 \%$ & 1.0471976 & $4.24 \times 10^{-14} \%$ \\
\hline$F_{11, \psi \mathrm{c}}$ & $F_{x}$ & -2.0943951 & -2.0939792 & $0.019 \%$ & -2.0943951 & $0 \%$ \\
\hline$F_{11, \psi s}$ & $F_{y}$ & -2.0943951 & -2.0939792 & $0.019 \%$ & -2.0943951 & $2.12 \times 10^{-14} \%$ \\
\hline$F_{11, \phi c}$ & $M_{x}$ & -1.0471976 & -1.0469896 & $0.019 \%$ & -1.0471976 & $2.12 \times 10^{-14} \%$ \\
\hline$F_{11, \phi s}$ & $M_{y}$ & -1.0471976 & -1.0469896 & $0.019 \%$ & -1.0471976 & $2.12 \times 10^{-14} \%$ \\
\hline
\end{tabular}

in the wall normal direction, $N_{p} \times N_{L}$ can become a major factor in the computational efficiency of the code. As shown in this section a non-uniform distribution of Lagrangian markers becomes unavoidable for computational efficiency.

\subsection{Area Weights}

In $§ 5$, a numerical technique was presented that specifies the area (and volume) weights of $N_{L}$ arbitrarily placed Lagrangian markers on a sphere. In this section, we will quantify the errors associated with these weights in the numerical evaluation of net force and moment due to various distributions of surface traction.

\subsubsection{Uniform Distribution of Lagrangian Markers}

First, we will consider the classic mathematical problem of uniform distribution of points on a sphere. Several algorithms can be found in the literature to distribute $N_{L}$ points on a sphere. According to Saff and Kuijlaars (1999), the spiral distribution of points that is presented in $\S 4$, shows reasonable agreement with the minimal energy theoretical estimates. Here we will evaluate the uniformity of this uniform distribution.

The spiral distribution method is used to place 1256 points equidistantly on a sphere of diameter $D=1$. For the first case, denoted as CASE1, equal area weights are assumed for all points, given by

$$
A_{l(1)}=\frac{\pi D^{2}}{N_{L}}=\frac{\pi}{1256}
$$

CASE2 has the area weights $A_{l(2)}$ computed using the method proposed in $\S 5$. Ten different force profiles are then imposed separately on the surface of the sphere. These profiles are defined by setting one of the ten coefficient of equation (5.5) to 1 while maintaining the rest at 0 . The resultant forces and moments of each profile are then evaluated numerically for CASE1 and CASE2 using (5.9) and the corresponding area weights of each case. The results are then compared to the analytical values given in (5.7) and (5.8). Each of these force distributions contribute to only a single component of net force or net moment (except $F_{00, r c}$ contributes neither to net force or net moment). Table 2 shows the analytical and numerical values of these non-zero resultants. Also included are the normalized error for CASE1 
and CASE2. A maximum error of about $0.2 \%$ is observed for CASE1 for all three components of force and the $z$-moment. Although this error can be considered small, it can limit overall accuracy in the context of high-order discretizations of the fluid flow. Furthermore, these errors correspond to the lowest order of spherical harmonic force distributions on the sphere. More complex force distributions are likely in the case of a sphere immersed in a more complex flow (such as in the presence of ambient turbulence). Importantly, the results demonstrate that even the best available uniform distribution for the Lagrangian markers, here using the spiral distribution of Saff and Kuijlaars (1997), involves significant error when assigned uniform weight. With a non-uniform weight distribution, as determined in CASE2, the error can be reduced to machine precision. In this case the rms variation in the weights of the 1256 markers, scaled by their average value is $2.67 \times 10^{-3}$. This approach provides a way to quantify the degree of uniformity of any algorithm for uniformly distributing markers on a sphere. Although not pursued here, the approach can be easily applied in an inverse manner to modify any near-uniform distribution of Lagrangian markers such that the errors become zero. Such a distribution of optimized markers can be described as the true uniform distribution of points on a sphere for any desired level of spherical harmonic approximation $(I$ and $P$ ).

It is of interest to study how well the uniform and non-uniform weights perform for higher-order spherical harmonic modes. Each combination of $(i, j)$ will be considered a spherical harmonic mode group and thus for a chosen value of $I$ there are $(I+1)(I+2) / 2$ mode groups. Here we will consider the top 15 mode groups corresponding to $I=4$. From the multipole expansion (see equation 5.3) it can be readily seen that each mode group includes up to six non-zero spherical harmonic coefficients and in this test each of these surface force distributions are turned by settling only these coefficients to one. Then, we first calculate the corresponding six net force and moment components analytically (define these to be $f_{i, E}$ for $\left.i=1,2, \ldots, 6\right)$, and compare the results with those from numerical integration using uniform and non-uniform weights (define these to be $f_{i, N}$ for $i=1,2, \ldots, 6$ ). Thus, for each mode group, 6 individual errors can be computed, from which a composite error measure is defined as the root mean square error (RMSE),

$$
R M S E=\sqrt{\frac{1}{6} \sum_{i=1}^{6}\left(f_{i, N}-f_{i, E}\right)^{2}} .
$$

The results are given in Figure 5. For CASE2 the error in the first three mode groups is very low at machine precision. This is expected since the derivation of the area weights in $\S 5$ uses these modes to force the numerical solution to be equal to their analytical value. For higher mode groups (i.e., $I>1$ ), the difference between CASE1 and CASE2 is reduced, since non-zero errors result even in the case of non-uniform weights. For the mode groups of $I=2$ and 3, the error is lower with the non-uniform weights. As we consider mode groups corresponding to $I=4$ and larger the errors in uniform and nonuniform weights become nearly identical. It should be noted, that all analytical resultant forces and moment are zero for modes greater than $(1,1)$. As for the accuracy of the higher order mode groups, it can be further improved by simply extending the method in $\S 5$ for $I=2$ or higher.

The error analysis is also performed for varying number of Lagrangian markers. The sphere is again subjected to different mode groups of force distribution and the area weights are calculated for both CASE1 and CASE2. Figure 6 shows the results for the first 3 mode groups. The error for CASE2 remains approximately constant at machine precision for all values of $N_{L}$ ranging from 10 to 2560 . Whereas, the error increases significantly for CASE1 as the number of Lagrangian markers decreases. It can also be seen that the error increases as the mode number increases. Figure 7 shows the error variation with increasing $N_{L}$ for select higher order mode groups for both CASE1 and CASE2. The behavior shown in Figure 5 for $N_{L}=1256$ seems to remain the same as the number of Lagrangian markers is varied. An approximate decay rate of error with increasing Lagrangian markers can be 


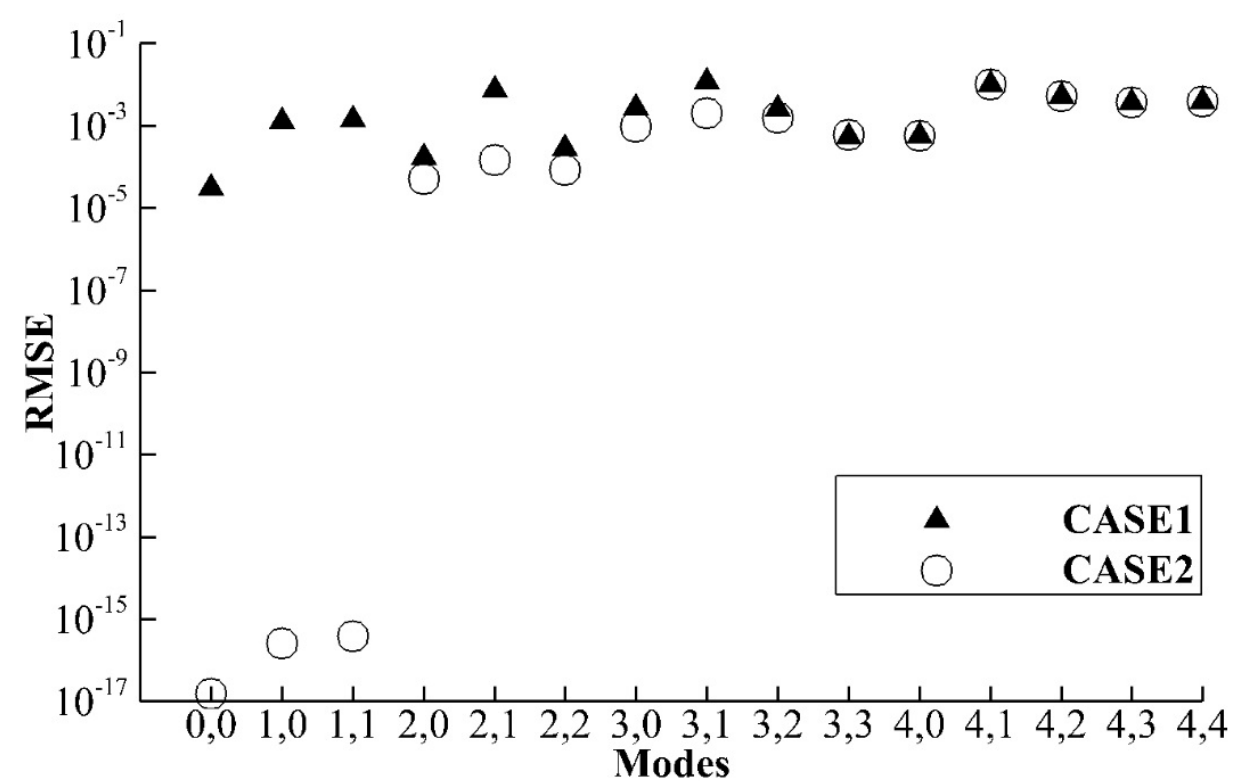

Figure 5. RMS error of the modes of the tractional force profile E for CASE1 and CASE2.

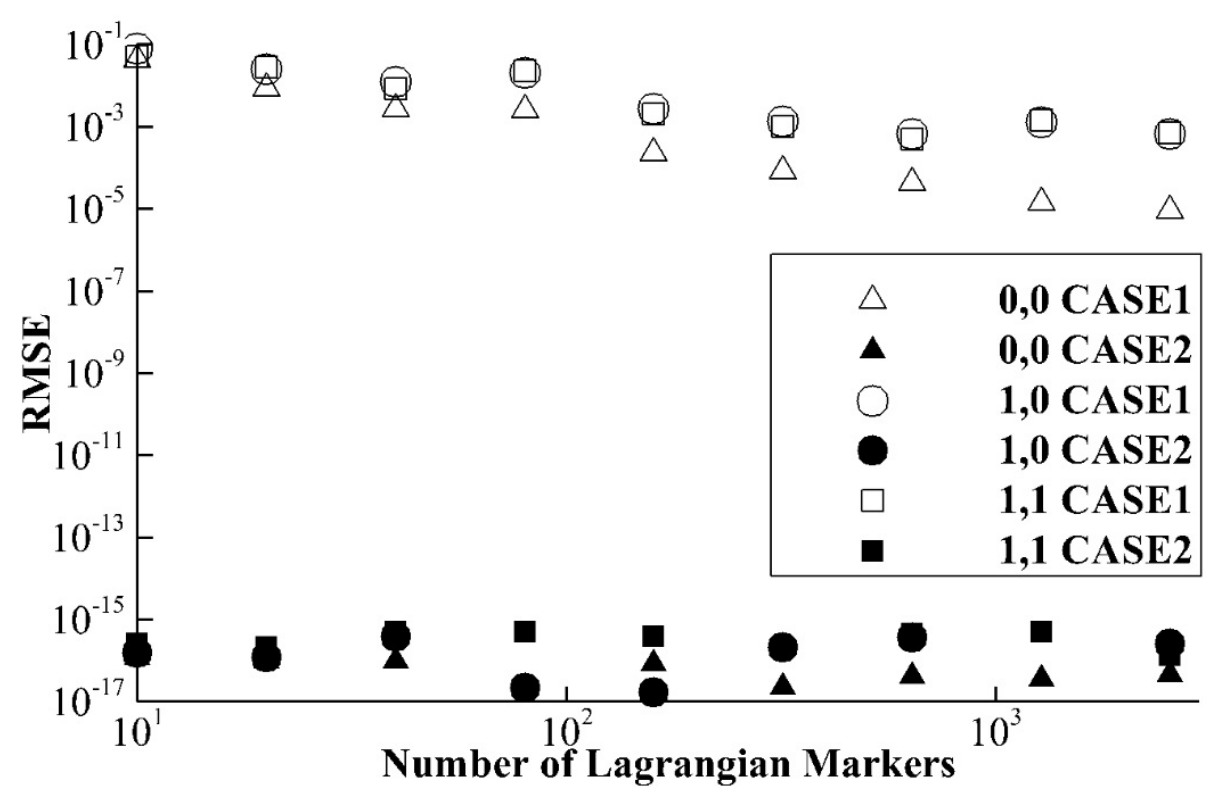

Figure 6. RMS error for increasing number of Lagrangian marker for the first 3 modes of the force profiles $\mathbf{E}$ for CASE1 and CASE2.

extracted from the data through a power law fit. For the modes shown in Figure 7, the decay rates are given in Table 3 for CASE1 and CASE2. For the lower 2 modes, CASE2 shows a faster decrease of the error as the number of Lagrangian points increase. The decay is almost identical for the higher 2 modes.

\subsubsection{Non-Uniform Distribution of Lagrangian Markers}

In this section, we extend the above analysis to Lagrangian markers that are distributed nonuniformly using the methods presented in $\S 4$. A sphere of diameter $D=1$ is placed on the wall of a channel of height 7.9, and 59 Eulerian grid points are used in the wall-normal direction. These 


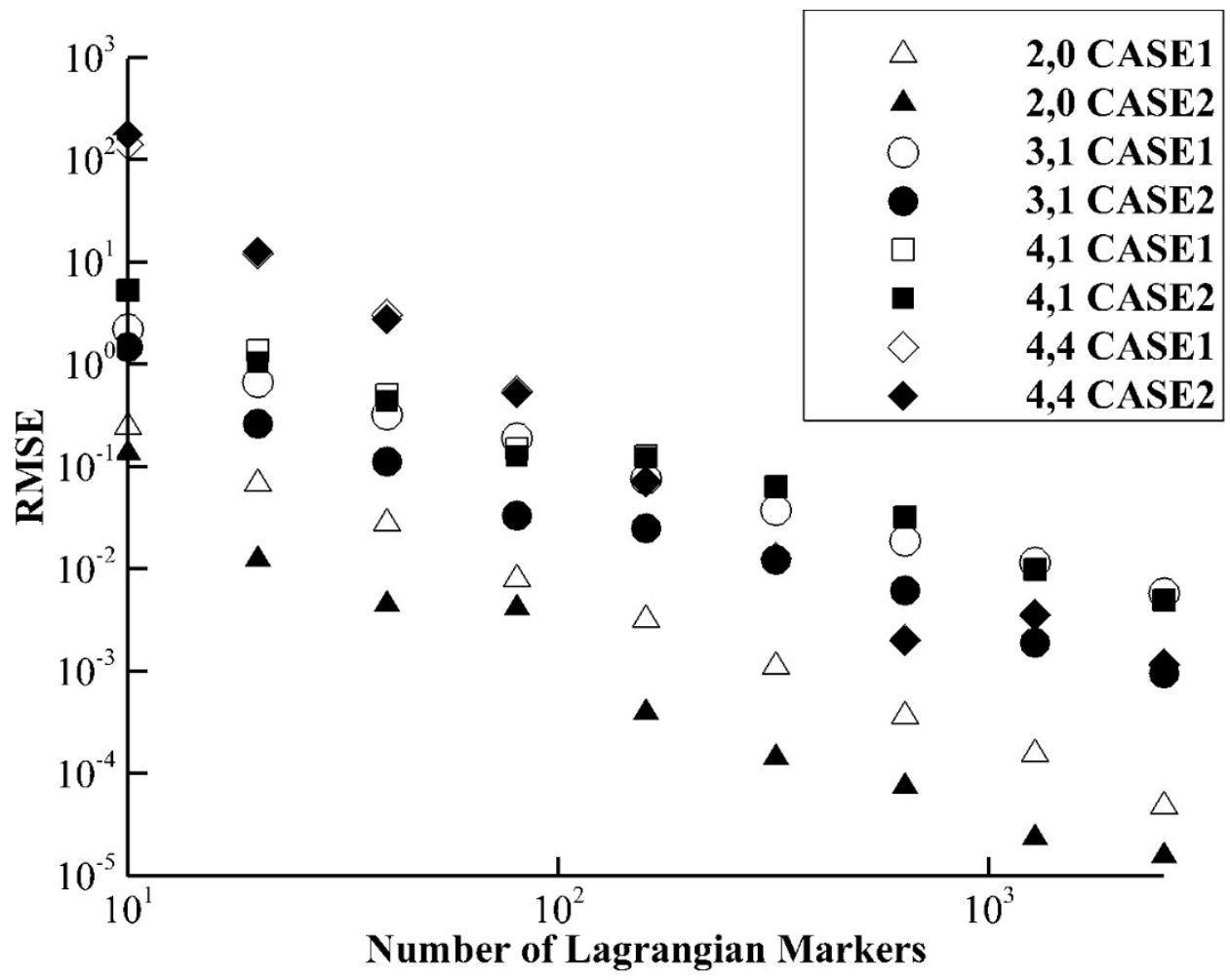

Figure 7. RMS error for increasing number of Lagrangian marker for select higher order modes of the force profiles $\mathbf{E}$ for CASE1 and CASE2.

Table 3. Decay rate of error with the Number of Lagrangian markers for CASE1 and CASE2.

\begin{tabular}{lll}
\hline Modes & CASE1 & CASE2 \\
\hline \hline 2,0 & $\sim N_{L}^{-1.517}$ & $\sim N_{L}^{-1.602}$ \\
3,1 & $\sim N_{L}^{-1.04}$ & $\sim N_{L}{ }^{1.227}$ \\
4,1 & $\sim N_{L}^{-1.179}$ & $\sim N_{L}^{-1.151}$ \\
4,4 & $\sim N_{L}^{-2.156}$ & $\sim N_{L}^{-2.175}$ \\
\hline
\end{tabular}

parameters are chosen because the resultant total number of Lagrangian markers from the methods in $\S 4$ is 1248 , which is comparable to the number of markers used in the previous section. Since the markers are non-uniformly distributed, it is no longer valid to assume equal area weights for all points. We define CASE3, where the area weights are assumed to be equal in each "subsection" of the sphere. The area weights can be calculated as,

$$
A_{l(3)}=\frac{\pi D^{2}}{N_{l}} .
$$

where $N_{l}$ is defined as,

$$
N_{l} \approx \frac{\pi}{3}\left(12 \frac{r_{c}^{2}}{\Delta z_{l}^{2}}+1\right),
$$

with $\Delta z_{l}$ the Eulerian grid spacing surrounding the subsection. For CASE4, the area weights are calculated with the non-uniform weight method of $\$ 5$. Table 4 shows the non-zero resultant forces and moments that arises from the nine lower-order modes. The table shows that as expected the non-uniform 
Table 4. Comparison of the analytical and numerical integration for CASE3 and CASE4 for non-zero values of resultant forces and moments.

\begin{tabular}{|c|c|c|c|c|c|c|}
\hline $\begin{array}{l}\text { Force } \\
\text { Profile }\end{array}$ & $\begin{array}{l}\text { Force/Moment } \\
\text { Direction }\end{array}$ & Analytical & CASE1 & $\%$ Error & CASE2 & $\%$ Error \\
\hline$\overline{F F_{10, r c}}$ & $F_{z}$ & 1.0471976 & 1.0530333 & $0.557 \%$ & 1.0471976 & $4.24 \times 10^{-14} \%$ \\
\hline$F_{11, r c}$ & $F_{x}$ & -1.0471976 & -1.0429718 & $0.404 \%$ & -1.0471976 & $4.24 \times 10^{-14} \%$ \\
\hline$F_{11, r s}$ & $F_{y}$ & -1.0471976 & -1.0390191 & $0.781 \%$ & -1.0471976 & $2.12 \times 10^{-14} \%$ \\
\hline$F_{10, \psi c}$ & $F_{z}$ & 2.0943951 & 2.0819909 & $0.592 \%$ & 2.0943951 & $0 \%$ \\
\hline$F_{10, \phi c}$ & $M_{z}$ & 1.0471976 & 1.0409955 & $0.592 \%$ & 1.0471976 & $2.12 \times 10^{-14} \%$ \\
\hline$F_{11, \mu \mathrm{c}}$ & $F_{x}$ & -2.0943951 & -2.0920525 & $0.112 \%$ & -2.0943951 & $2.12 \times 10^{-14} \%$ \\
\hline$F_{11, \psi s}$ & $F_{y}$ & -2.0943951 & -2.0960051 & $0.077 \%$ & -2.0943951 & $2.12 \times 10^{-14} \%$ \\
\hline$F_{11, \phi c}$ & $M_{x}$ & -1.0471976 & -1.0460262 & $0.112 \%$ & -1.0471976 & $2.12 \times 10^{-14} \%$ \\
\hline$F_{11, \phi s}$ & $M_{y}$ & -1.0471976 & -1.0480026 & $0.077 \%$ & -1.0471976 & $2.12 \times 10^{-14} \%$ \\
\hline
\end{tabular}

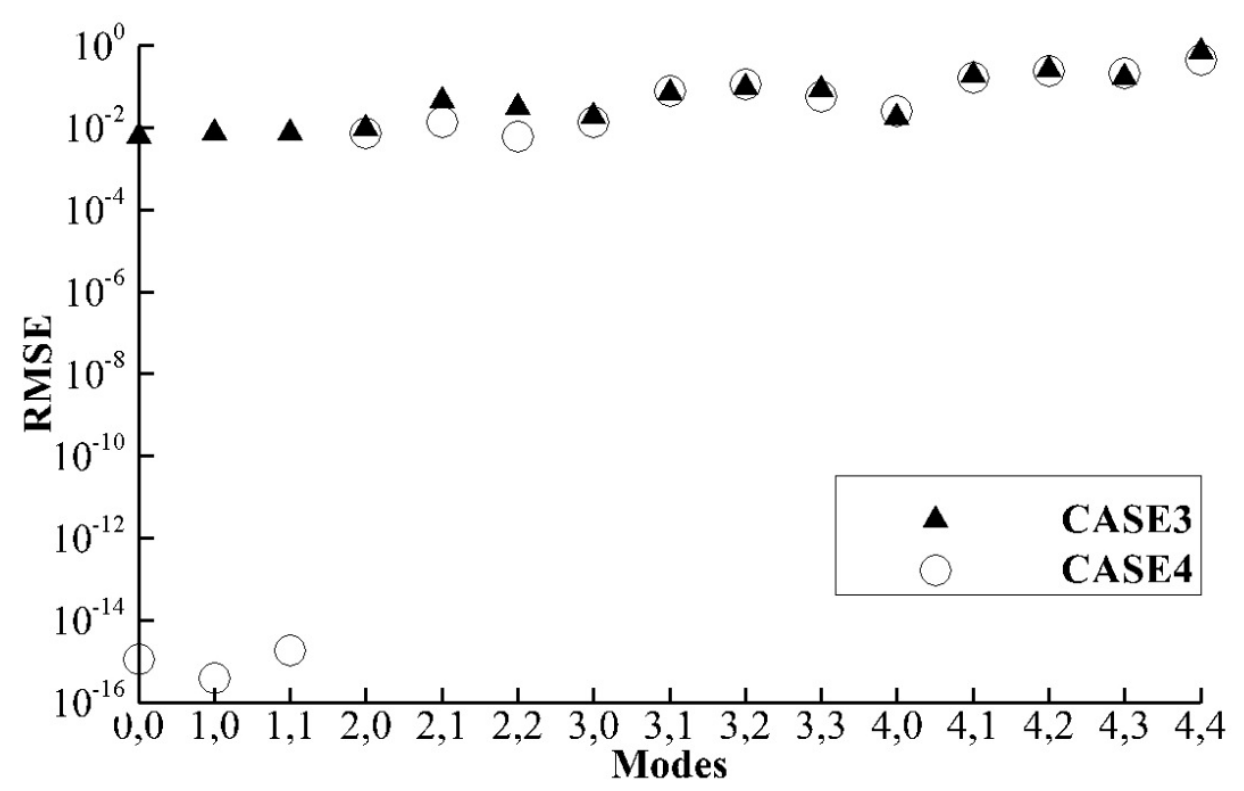

Figure 8. RMS error of the modes of the tractional force profile E for CASE3 and CASE4.

weights determined using the spherical harmonics results in machine precision. Upon, comparing the values of CASE3 and the ones of CASE1 from Table 2, it can be seen that the assumption of equal weights within subsections results in larger errors for a non-uniform distribution. Therefore, the use of non-uniform area weights defined in this study becomes even more beneficial when Lagrangian markers are non-uniformly distributed on the surface of the sphere.

The RMSE for the top fifteen mode groups are shown in Figure 8 for CASE3 and CASE4. Similar to the uniform distribution case, the RMSE for the first three modes are nearly zero for CASE4. For the next 4 mode groups, the results for CASE3 and CASE4 are much closer to each other, but the RMSE for CASE4 is systematically smaller than CASE3. For the modes greater than $(3,0)$, RMSE of both cases are comparable, taking into account the increased noise arising from the spherical harmonic expansions for high modes. 


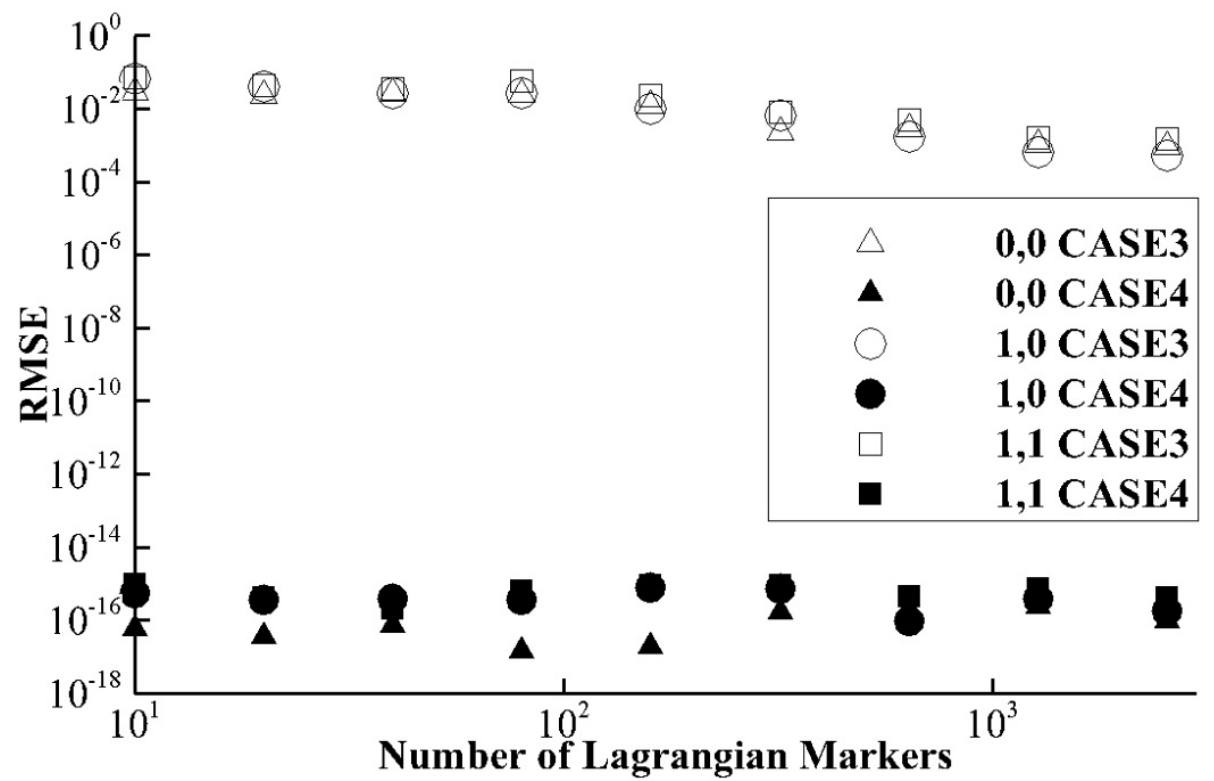

Figure 9. RMS error for increasing number of Lagrangian marker for the first 3 modes of the force profiles $\mathbf{E}$ for CASE3 and CASE4.

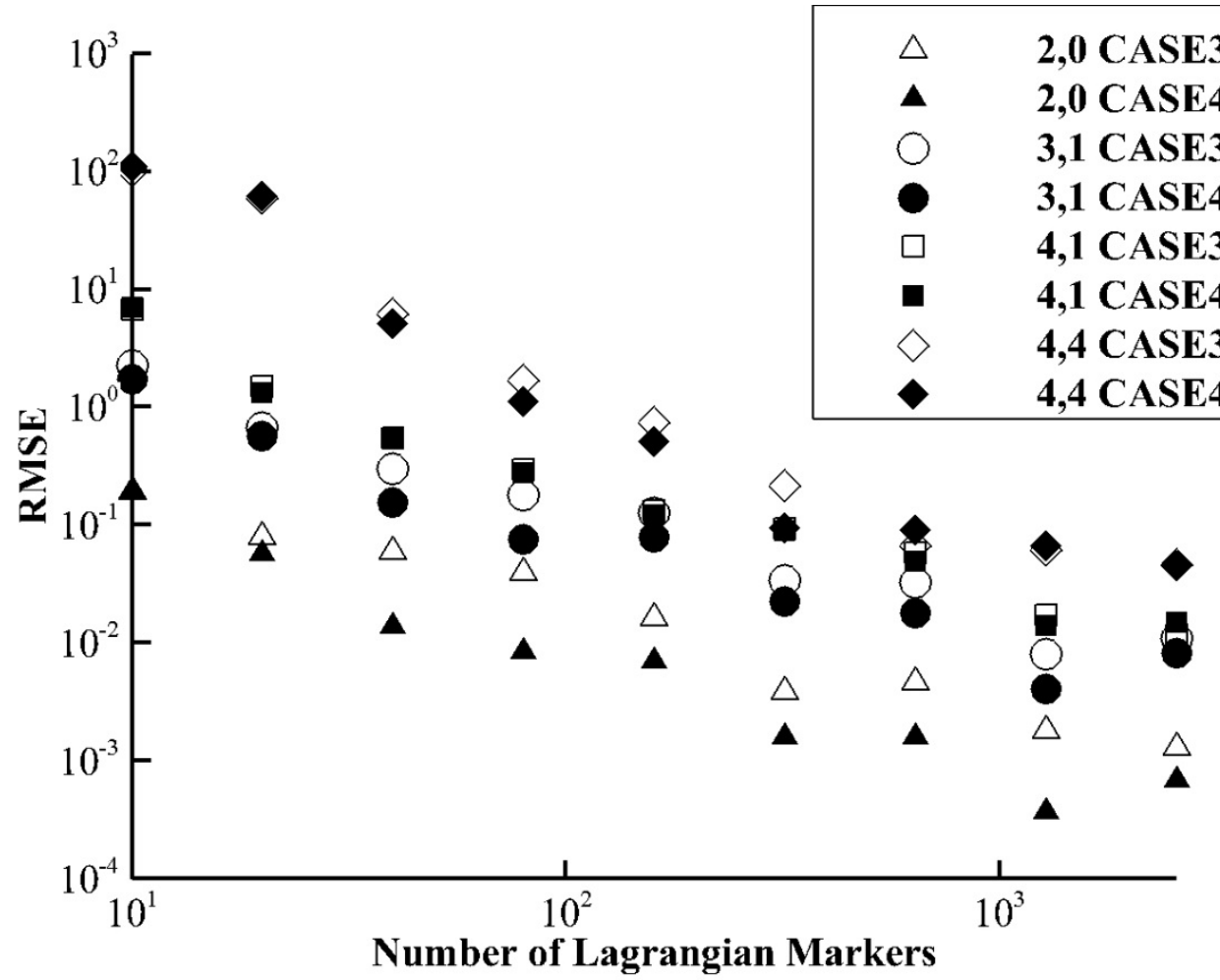

Figure 10. RMS error for increasing number of Lagrangian marker for select higher order modes of the force profiles $\mathbf{E}$ for CASE3 and CASE4.

The effect of the number of Lagrangian markers distributed non-uniformly on a sphere is shown in Figure 9 for the first three mode groups, and in Figure 10 for select higher mode groups. The results are consistent with those obtained for uniform distribution. For mode groups $(2,0)$ and $(3,1)$, CASE4 shows 


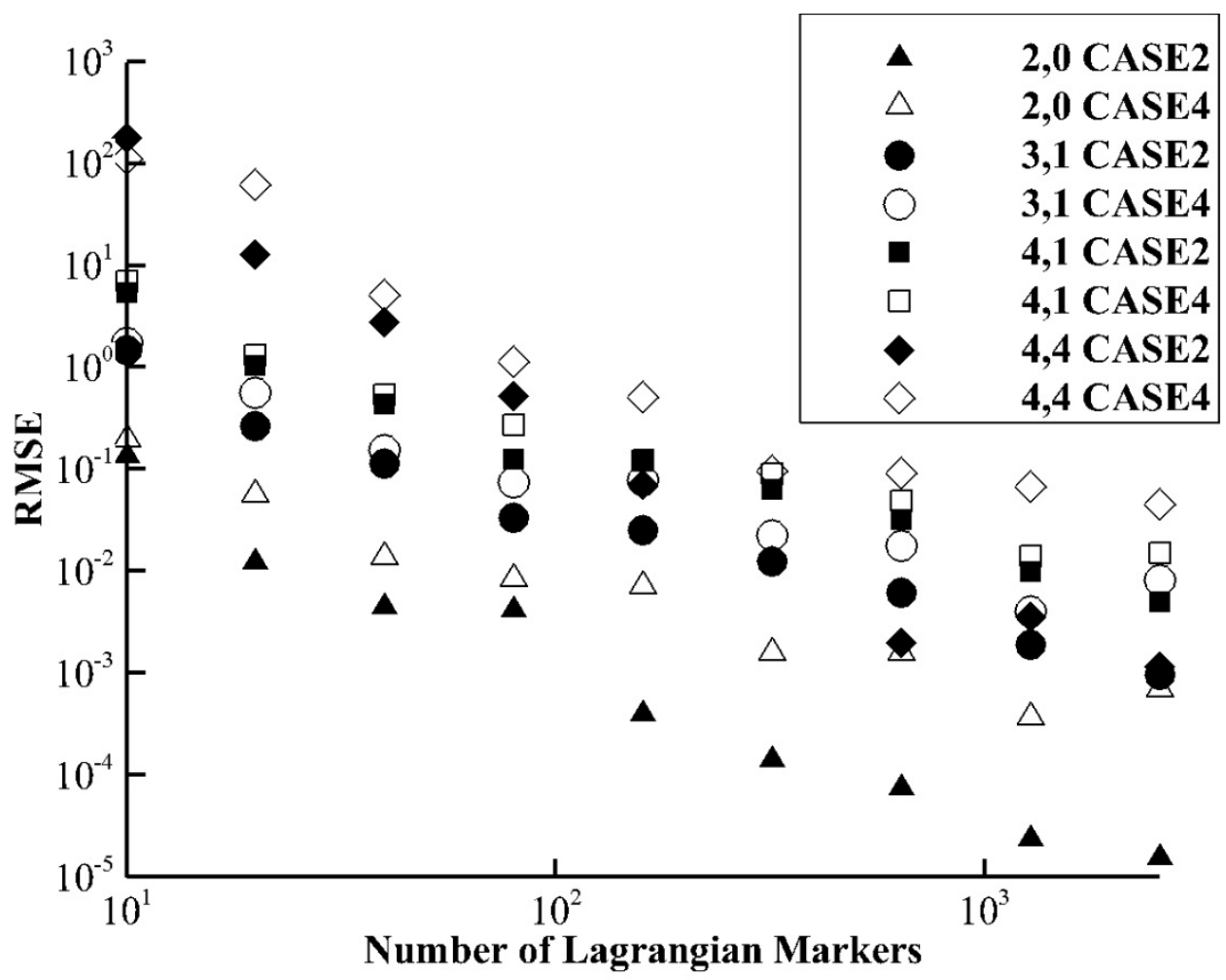

Figure 11. RMS error for increasing number of Lagrangian marker for select higher order modes of the force profiles $\boldsymbol{E}$ for CASE2 and CASE4.

a faster reduction in error than CASE3 as the number of Lagrangian markers increases, but shows no change for mode groups $(4,1)$ and $(4,4)$.

It is interesting to compare CASE2 and CASE4. Both use the method presented in $\S 5$ for the evaluation of non-uniform area weights. CASE2 uses a near uniform distribution of markers while CASE4 employs a non-uniform distribution. The RMSE values for select mode groups are shown in Figure 11. CASE2 shows a significantly lower error for all mode groups. Also, the slope of a power-law fit is significantly smaller for CASE2, meaning that the error decreases at a faster rate as the number of Lagrangian points increases for the uniform case. This clearly suggests that a non-uniform distribution of Lagrangian markers is preferred only when there is benefit in terms of computational efficiency over a corresponding uniform distribution of markers.

Note that the way the markers are non-uniformly distributed in this case (uniformly within subsections) made it possible to have an algorithm to determining equal weights in each subsection. But in case where the non-uniform distribution of Lagrangian markers is determined by other methods or perhaps even randomly, there may not be a ready approximation for the area weights. In such cases the present method would provide a good technique to obtain the area weights.

\subsection{Sphere in a Channel Flow}

In this section we will test the performance of the above described immersed boundary numerical methodology that employs a non-uniform distribution of Lagrangian markers in a non-uniform Eulerian grid. Two different problems of a channel flow over a stationary particle and freely moving particle will 

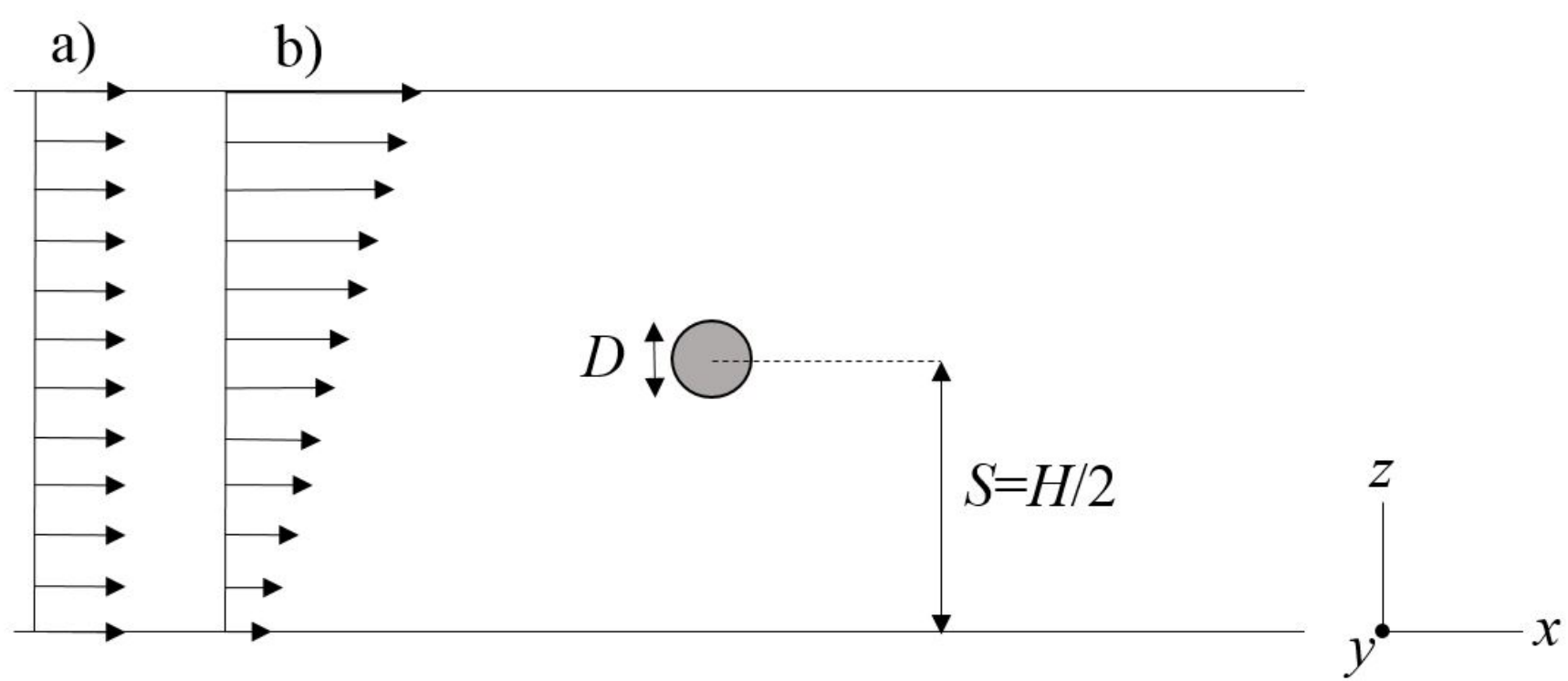

Figure 12. Schematic of the geometry used in $\S 6.3 .1$ and $\S 6.3 .2$ for the a) uniform and b) shear ambient flow.

be considered. In each case the results will be compared against other simulation results that have been already established in the literature.

\subsubsection{Fixed Sphere}

A sphere of diameter $D=1$ is placed at the center of a channel with dimensions of $50 \times 14 \times 8$ along the streamwise, spanwise and wall-normal directions. A schematic of the geometry is shown in Figure 12. The mesh size used is $750 \times 210 \times 151$. This leads to a sphere resolution of 15 grid points per diameter in the streamwise and spanwise direction. As for the wall-normal direction, the sphere resolution depends on its location in the channel and would range from 35 grid point per diameter for a sphere near the wall, to 13 grid points per diameter for a sphere at the center. The generation of Lagrangian markers is completed using the method outlined in $\S 4$, which results in 450 non-uniformly distributed markers. The weights are assigned following the method presented in $\S 5$. The length in the streamwise direction was chosen to be large to reduce the effects of streamwise periodicity. This would allow a reasonable comparison of the hydrodynamic forces with other studies which specify an inflow velocity rather than a periodic boundary.

The sphere is held fixed in an ambient flow of unit velocity magnitude driven by specifying a nonzero streamwise velocity for the walls and enforcing the no-slip boundary condition. The force coefficient $C_{F}$ is defined as,

$$
C_{F}=\frac{F}{\frac{1}{8} \rho_{f}\left|u_{r}\right|^{2} \pi D^{2}} .
$$

The drag coefficients for different Reynolds numbers are reported in Figure 13. The results are shown alongside the standard drag law by Schiller and Naumann (1933),

$$
C_{D}=\frac{24}{R e}\left(1+0.15 \operatorname{Re}^{0.687}\right) \text {. }
$$




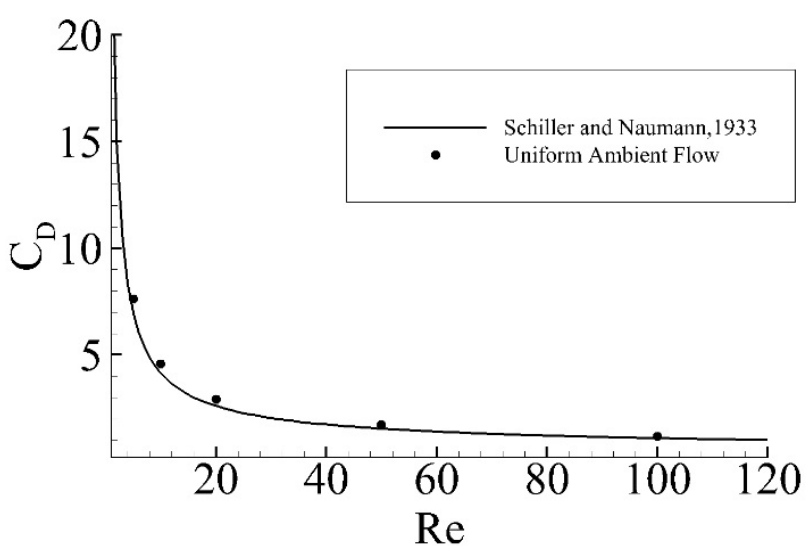

Figure 13. Variation of the coefficient of drag for different Reynolds number in a uniform ambient flow for the present numerical simulations and the prediction by Schiller and Naumann (1933).

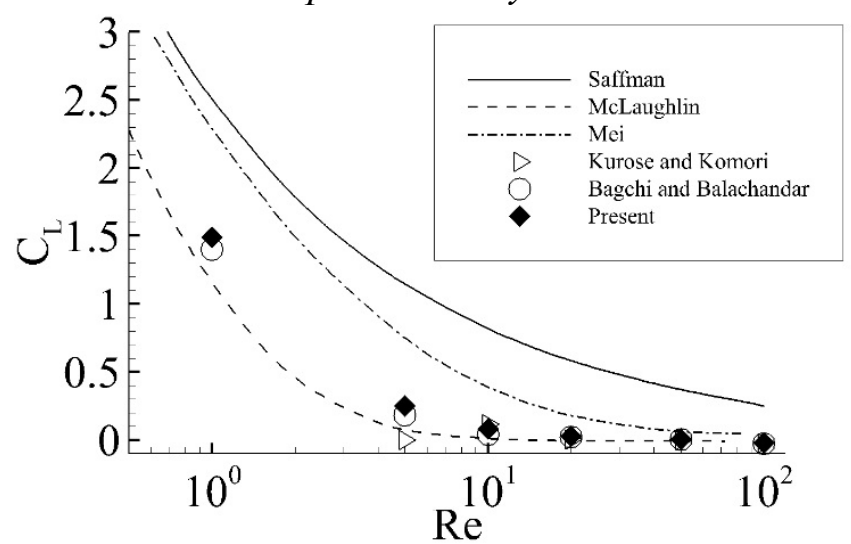

Figure 14. Coefficient of lift for a sphere in a steady shear flow for different Reynolds numbers $(G=0.4)$. The domain for $R e=1$ is $20 \times 21 \times 20$ with a mesh size of $300 \times 314 \times 377$. For all other Reynolds numbers, the domain is $50 \times 14 \times 8$ and the mesh size used is $750 \times 210 \times 151$.

The data are in reasonable agreement. The simulations show a slightly higher drag and this can be attributed to the confining effect of the lateral flat boundaries and to the imposed periodicity in the streamwise direction.

For additional validation we will consider a linear shear flow. The undisturbed ambient velocity profile is defined as,

$$
u_{x}=u_{b}+G\left(z-z_{b}\right), \quad u_{y}=0, \quad u_{z}=0 .
$$

$u_{b}$ and $z_{b}$ are the velocity and height of the lower wall respectively and $G$ is the shear rate. The steady lift coefficient for a nonrotating sphere in a linear ambient shear flow of $G=0.4$ are plotted in Figure 14 . The theoretical predictions by Saffman (1965) and McLauglin (1991), the approximate expression by Mei (1992), and numerical simulations data from Kurose and Komori (1999) and Bagchi and Balachandar (2002) are also plotted. The present numerical results are in good agreement with the other two numerical simulations. It should be noted that the numerical simulations by Kurose and Komori (1999) and Bagchi and Balachandar (2002) employed finite difference and spectral methodologies, with body-fitted grids resolving the sphere. 


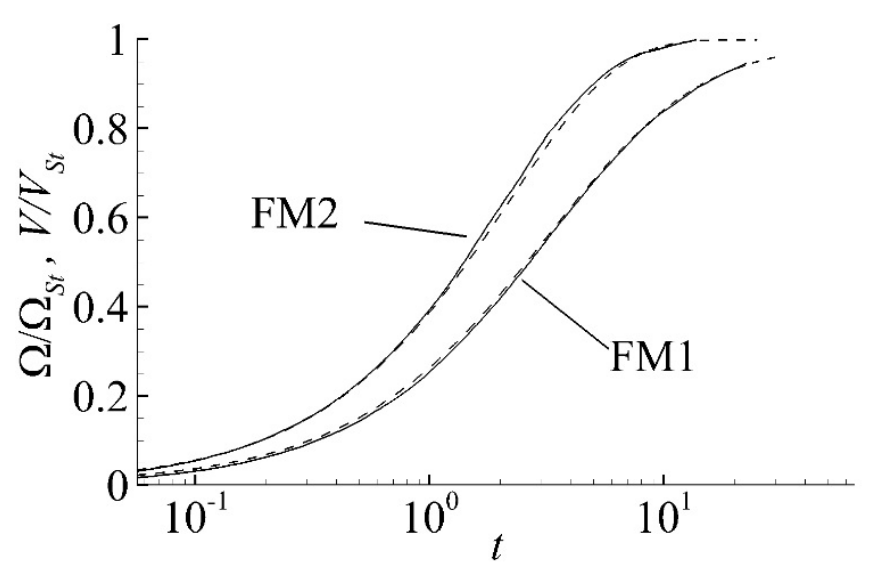

Figure 15. Transient behavior of the translational (FM1) and rotational (FM2) case for the (-) present method and (---) Bagchi and Balchandar (2002).

Table 5. Torque-free rotation rate of a sphere in an ambient shear flow.

\begin{tabular}{llll}
\hline$R e$ & Bagchi and Balachandar & Present $\Omega_{s t} / \Omega_{f}$ & \% difference \\
\hline \hline 3.75 & 0.8722 & 0.8834 & $1.28 \%$ \\
9.83 & 0.7864 & 0.7800 & $0.82 \%$ \\
50 & 0.5523 & 0.5420 & $1.87 \%$ \\
\hline
\end{tabular}

\subsubsection{Free Motion of a Sphere in a Channel Flow}

A sphere of diameter $D=1$ is placed at the center of a channel with dimensions of $50 \times 14 \times 8$ and a mesh of $500 \times 140 \times 91$. The sphere is allowed to rotate and translate freely under the action of the hydrodynamic forces. The equations of motion that governs the sphere's movement are simultaneously solved along with the flow. The discretized version of the three-stage Runge-Kutta scheme used in our simulations involving moving particles can be found in Uhlmann (2005). The equations for the translational velocity $U_{c}$, angular velocity $\Omega_{c}$ and the spatial location of the sphere's center $\boldsymbol{X}_{c}$ are given by,

$$
\begin{aligned}
& \frac{\boldsymbol{U}_{c}^{m+1}-\boldsymbol{U}_{c}^{m}}{\Delta t}=-\frac{\rho_{f}}{V_{c}\left(\rho_{p}-\rho_{f}\right)} \sum_{l=0}^{N_{L}} F_{\left(X_{l}\right)} \Delta V_{l}+2 \frac{c d_{m}}{\Delta t} \boldsymbol{g} \\
& \frac{\boldsymbol{\Omega}_{c}^{m+1}-\boldsymbol{\Omega}_{c}^{m}}{\Delta t}=-\frac{\rho_{f}}{\left(\rho_{p}-\rho_{f}\right)} \frac{1}{\left(I_{c} / \rho_{p}\right)} \sum_{l=0}^{N_{L}}\left(\boldsymbol{X}_{l}-\boldsymbol{X}_{c}\right) \times F_{\left(X_{l}\right)} \Delta V_{l} \\
& \frac{\boldsymbol{X}_{c}^{m+1}-\boldsymbol{X}_{c}^{m}}{\Delta t}=\frac{c d_{m}}{\Delta t}\left(\boldsymbol{U}_{c}^{m+1}-\boldsymbol{U}_{c}^{m}\right)
\end{aligned}
$$

where $\rho_{p}, \rho_{f}$, and $V_{c}$ are the density of the sphere, density of the fluid and total volume of the sphere respectively. $F$ is the Lagrangian forcing term obtained from equation (2.11) using the fluid velocity field from the previous time step (or Runge-Kutta stage in this case).

In the first simulation, denoted by FM1, a sphere of density $\rho_{p}=5 \rho_{f}$ is held fixed in a uniform ambient flow until a steady state is achieved. The sphere is then released to translate freely downstream. The freedom of rotation was not allowed in this case. The sphere reaches steady translational velocity $V_{s t}$ when the drag force approaches zero. In the second simulation, denoted by FM2, the sphere is placed in 


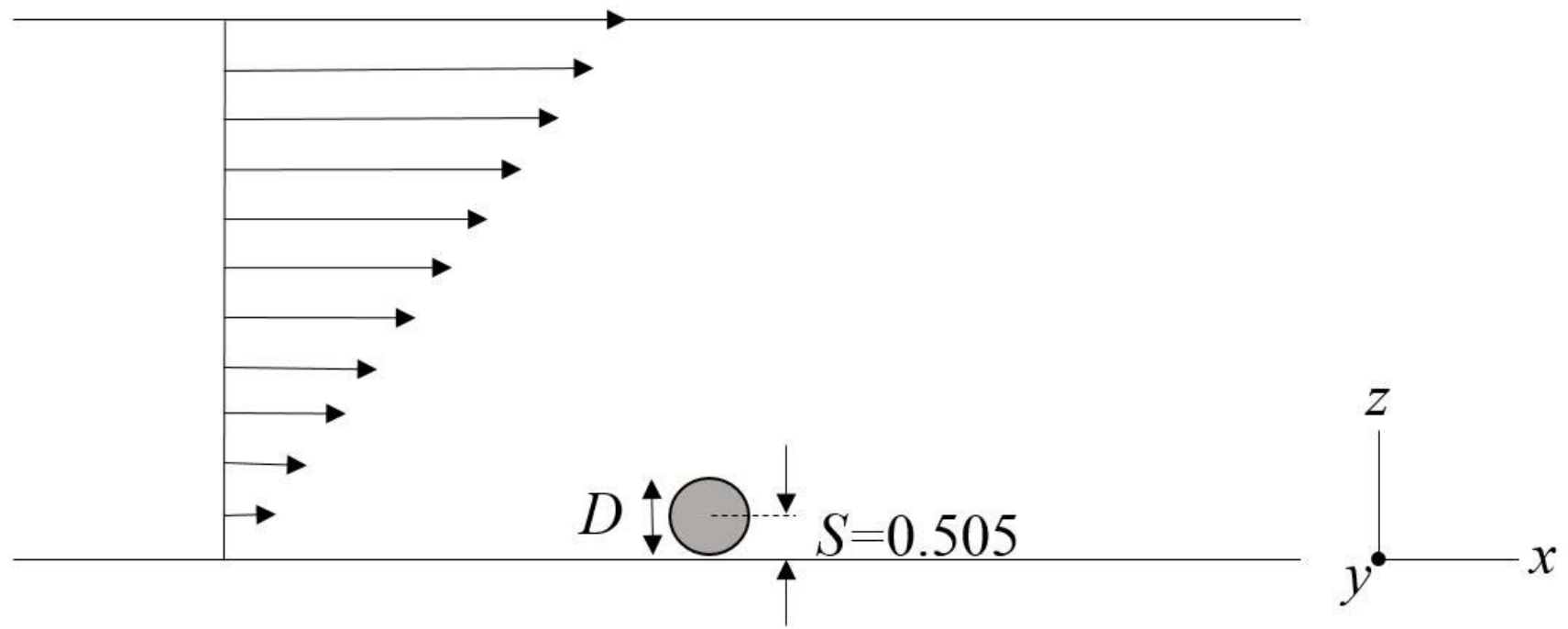

Figure 16. Schematic of the geometry used in $\S 6.3 .3$.

a steady ambient shear flow where $G=0.15$. Similar to FM1, the sphere is held fixed until a steady state torque is achieved. Free rotation is then permitted without the ability to translate downstream. When the torque approaches 0 , the sphere is said to have reached a steady state angular velocity of $\Omega_{s t}$. A moderate Reynolds number of $R e=20$ is chosen.

Figure 15 shows the transient phase during the translational and rotational motion of the sphere in cases FM1 and FM2, respectively. The data is normalized by the steady state translational and angular velocities respectively. The present simulations match very closely the numerical data from Bagchi and Balachandar (2002) for both cases. Bagchi and Balachandar (2002) also present a relation for the steady state angular velocity in terms of the Reynolds number as

$$
\begin{aligned}
\frac{\Omega_{s t}}{\Omega_{f}} & =1-0.0364 R e^{0.95}, \quad \text { for } 0.5<R e \leq 5, \\
& =1-0.0755 R e^{0.455}, \quad \text { for } 5<R e \leq 200 .
\end{aligned}
$$

where $\Omega_{f}$ is the ambient rotation rate defined as $\Omega_{f}=G / 2$. Three Reynolds numbers were tested with the present numerical technique, covering both regimes of equation (6.13). The results are presented in Table 5. A maximum difference of $1.87 \%$ at a Reynolds number of 50 is observed.

\subsubsection{Sphere near the Channel Wall}

In this test a sphere of diameter $D=1$ is placed in a channel with dimensions of $50 \times 14 \times 8.505$ such that it is close to the channel wall, with its center at a non-dimensional distance of $S=0.505$ from the channel wall. A schematic of the geometry is shown in Figure 16. In this example the sphere is nearly in contact with the wall. The Eulerian mesh used is $1000 \times 280 \times 121$. Using the non-uniform distribution algorithm presented in $\S 4$, we obtain 2968 Lagrangian markers. This is two orders of magnitude lower than 164509 Lagrangian marker that would be generated if a uniform Lagrangian distribution was used with the smallest mesh size surrounding the grid taken as a criterion in equation (4.1). 
a)

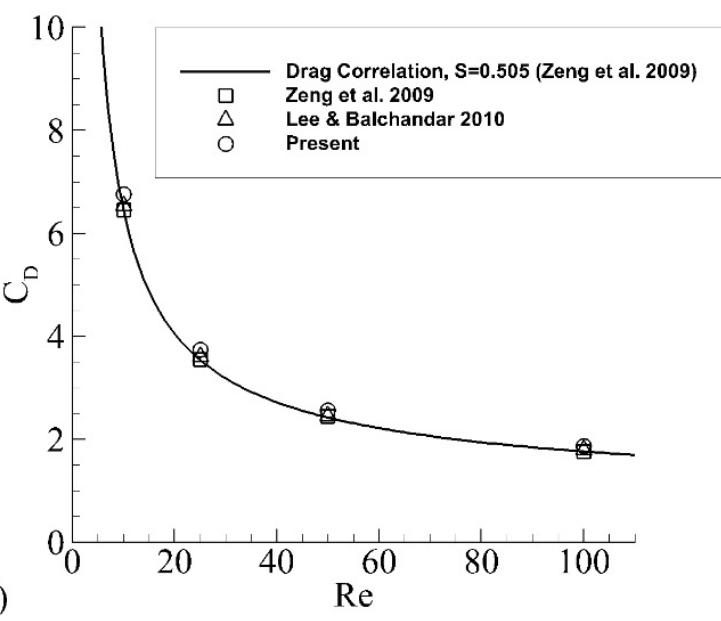

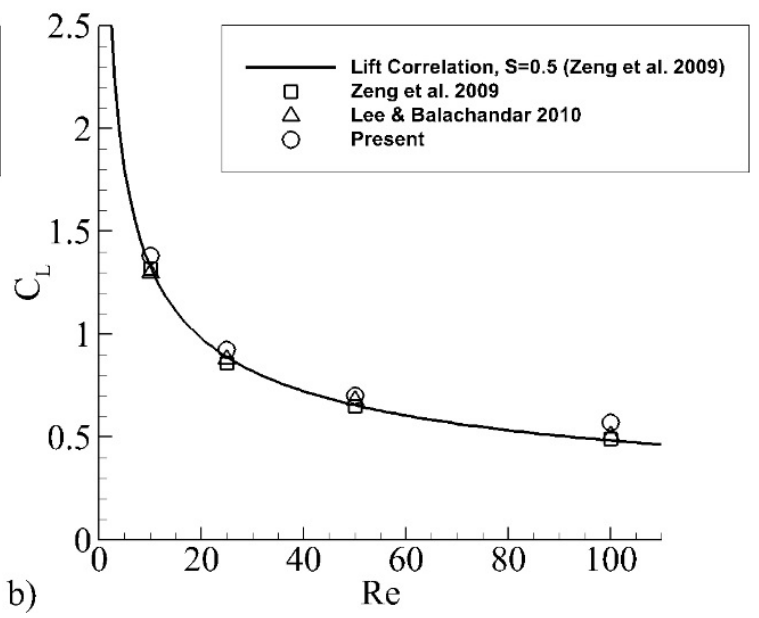

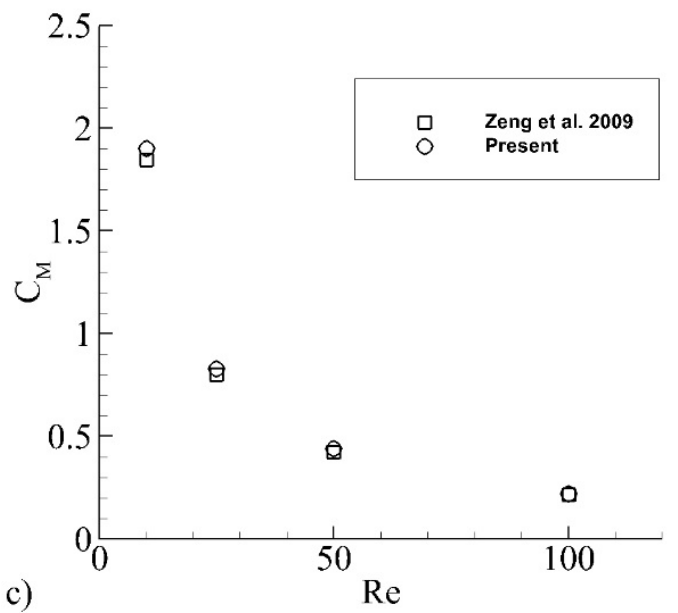

Figure 17. Coefficient of a) drag, b) lift and c) moment for a sphere at a distance $S=0.505$ from the wall in an ambient shear flow.

Table 6. Comparison of the coefficients of drag, lift and moment of the present method and of the uniform spacing of Lagrangian points in a non-uniform Eulerian grid. $($ Re $=50)$

\begin{tabular}{lllll}
\hline & $N_{L}$ & $C_{D}$ & $C_{L}$ & $C_{M}$ \\
\hline \hline Zeng et. al (2008) & - & 2.44 & 0.65 & 0.42 \\
Lee \&Balachandar (2010) & 5,028 & & & \\
& & 2.45 & 0.68 & \\
Non-Uniform Distribution & 1,699 & & & \\
& 2,968 & 2.65 & 0.84 & 0.46 \\
& 4,621 & 2.59 & 0.74 & 0.43 \\
Uniform Distribution & & 2.55 & 0.70 & \\
& 52,059 & & & 0.42 \\
& 164,458 & 2.65 & 0.70 & 0.39 \\
& 144,598 & 2.61 & 0.56 & 0.38 \\
\hline
\end{tabular}



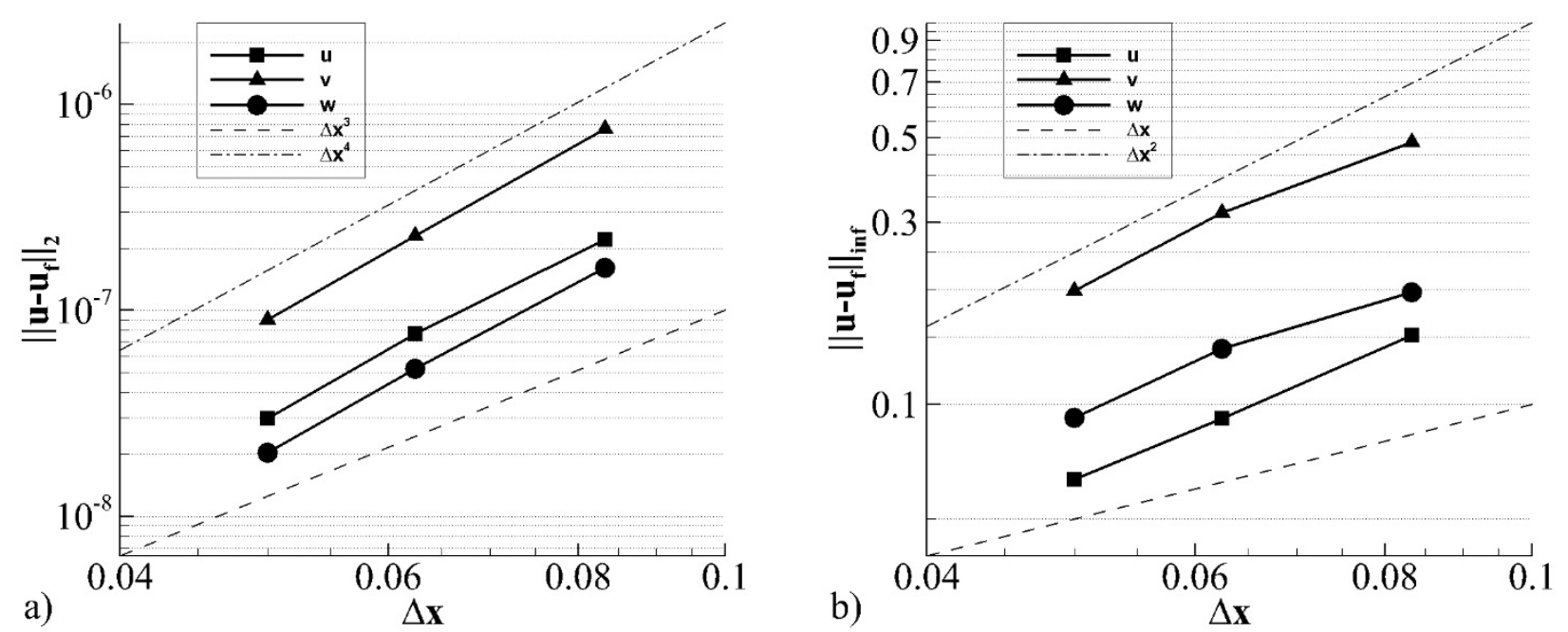

Figure 18. Log-log plot showing the convergence of the a) $L_{2}$ and b) $L_{\infty}$ norm of the error.

The sphere is held fixed in an ambient shear flow of $R e=10,25,50$ and 100. The coefficient of drag, lift and moment for the different Reynolds numbers are shown in Figure 17 with the results from Zeng et al (2009) and Lee \& Balchandar (2010). The drag and lift correlation for a sphere near the wall from Zeng et al (2009) is plotted alongside in Figure 17a and Figure 17b for comparison. The results are in good agreement with the other two studies. Zeng et al (2009) uses spectral elements with a bodyfitted grid. Lee \& Balachandar (2010) uses IBM with a uniform Eulerian grid in the vicinity of the sphere which was resolved with 5028 Lagrangian marker. This is $69 \%$ more than the number of points used in the present simulations. The advantage will be even more when simulating spheres in a turbulent boundary layer, as in Zeng et al. (2008). In which case one will need either a non-uniform grid close to the wall or a very fine uniform grid. In either approach, without an algorithm for non-uniform distribution of Lagrangian markers and associated weights, computational expense can be severe.

Another set of simulations for $R e=50$ are conducted for the case of a sphere located close to the channel wall at $S=0.505$. In this test, we use 3 different mesh sizes of $600 \times 168 \times 91,800 \times 224 \times 121$, and $1000 \times 280 \times 151$. The streamwise and spanwise sphere resolution are 12,16 , and 20 points per diameter respectively. As for the wall-normal direction the resolution is 19, 25, and 31 points per diameter. Upon distributing the Lagrangian points non-uniformly, we obtain 1699, 2968, and 4621 Lagrangian markers respectively. The same simulations are repeated with a uniform distribution of the Lagrangian markers, taking the smallest Eulerian grid surrounding the sphere as $h$ in equation (4.1). The results are shown in Table 6. It should be noted that although the Eulerian mesh is finer in the third case, the number of Lagrangian points for the uniform distribution is smaller. This is due to the fact that the small gap of 0.005 between the sphere and the wall puts the sphere between the third and the fourth boundary node for the third mesh size; whereas, it is lying between the second and the third boundary node for the second mesh size. This shows the abrupt jump in the number of uniform Lagrangian markers that can result from a small change in the location of the sphere, which would lead to significant numerical noise, especially for a moving sphere. The method proposed in $\$ 4$ ensures a smoother transition. The results presented in Table 6 shows a monotonic convergence of the coefficients of drag, lift and moment. The converged values of the non-uniform case compare well with the ones reported by Zeng et al. (2008) and Lee \& Balachandar (2010) which are presented alongside for reference. As for the uniform case, there is a significant discrepancy in the coefficients of lift and moment. The Lagrangian spacing on the 
upper end of the sphere is much finer than the surrounding Eulerian mesh in the uniform distribution case. The presence of even a small error in the calculation of the Lagrangian forcing or the Lagrangian weights would lead to a much larger error in the total resultant force.

A fourth simulation using a finer mesh of $420 \times 168 \times 91$ is conducted. This results in 10811 nonuniformly distributed Lagrangian markers. The $\mathrm{L}_{2}$ and $\mathrm{L}_{\infty}$ norm of the error are calculated for the first 3 mesh sizes, considering the fourth mesh size results to be most accurate. The mesh size for the 3 cases are $\Delta x=\Delta y=1 / 12,1 / 16$, and 1/20. The results are shown in Figure 18 for the three components of velocity. The L2 norm shows a convergence of $\Delta x^{4}$ and the $\mathrm{L}_{\infty}$ norm shows a convergence of $\Delta x^{2}$. The lower convergence rate of $\mathrm{L}_{\infty}$ is the direct result of the second order IBM scheme used.

\subsubsection{Pressure Driven Channel Flow Through a Cluster of Monodisperse Particles}

As further demonstration, here we consider 640 spheres of diameter $D=1$ are randomly distributed in a channel with streamwise, spanwise and wall-normal dimensions of $3 \pi \times 3 \pi \times 6 \pi$. This configuration can represent a porous medium with a volume fraction of 0.2. A Chebychev Gauss-Lobatto mesh of $192 \times 192 \times 401$ is employed. Figure 19 shows a schematic of the channel with the random distribution of particles. It clearly shows the higher density of Lagrangian markers for the particles close to the top and bottom no-slip walls. Periodic boundary conditions are used along the streamwise and spanwsise directions. The total number of Largrangian markers used is 920,222 compared to 3,994,997 if a uniform distribution was used, which is a $76.97 \%$ reduction.

The flow is driven by imposing a unit non-dimensional constant pressure gradient along the streamwise direction. Figure 20 shows the streamwise velocity contour plot on the spanwise center plane at steady state. Figure 21 shows the planar average streamwise velocity variation in the wall-normal direction. The particle Reynolds number is $\operatorname{Re}_{b}=4.47$, and it is based on the bulk velocity in the channel at steady state and the particle diameter. Even for this relatively low Reynolds number, the need for a higher Eulerian grid resolution near the walls is evident due to the sharp velocity gradients.

The same simulation is repeated, this time with no stress boundary conditions at the walls. This would allow for the total pressure drop to be balanced by the drag on the spheres only. A simple force balance equation would be,

$$
F_{D}=\left(\frac{\Delta p}{\Delta x} L_{x}\right) L_{y} L_{z}
$$

where $F_{D}$ is the total drag force on the spheres. The total drag computed from the Lagrangian forcing using equation (2.11) is 1676.6. This value is compared to the exact force obtained from the right hand side of equation (6.14), which in the present simulation is 1674.34 , a difference of $0.135 \%$ from the drag computed through Lagrangian forcing. This provides additional support for the force calculation using non-uniform distribution of Lagrangian markers.

\section{Conclusion}

In this paper, an efficient method to simulate a sphere in a channel flow using immersed boundary methods was presented. For a non-uniform Eulerian grid, an algorithm for non-uniform distribution of Lagrangian points on a sphere is presented. The Lagrangian markers representing the sphere are generated to match the surrounding Eulerian grid, depending on the sphere's position within the channel. The results shows a significant reduction in Lagrangian markers without the loss of information provided by the Eulerian grid. 


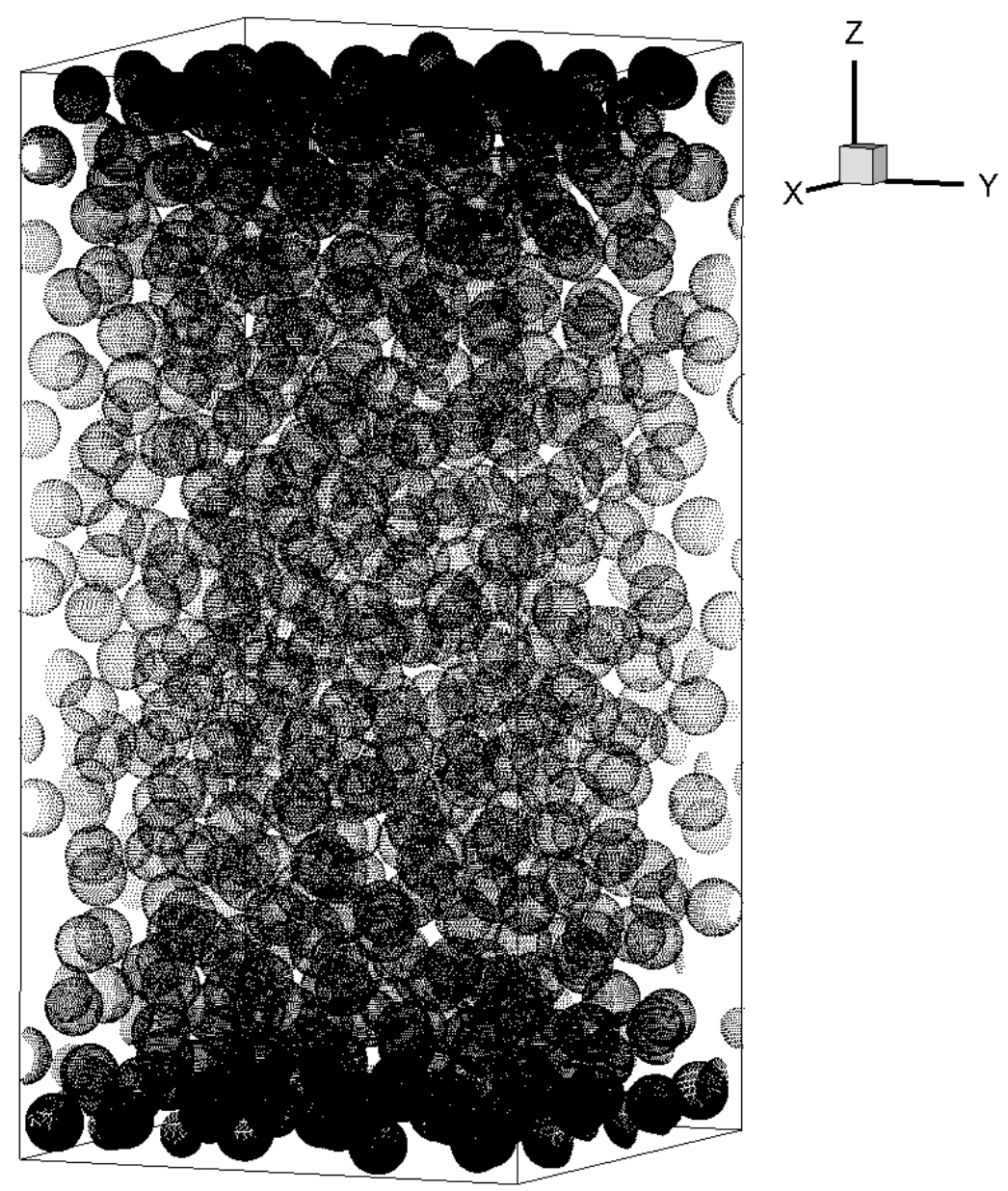

Figure 19. Schematic of the distribution of the Lagrangian markers for 640 monodisperse spherical particles in a periodic channel.

Also, a new numerical technique to assign area weights for a random number of points on a sphere was presented. The weights are used in the spreading step of the IBM. In addition to the significance of the proposed technique for the IBM, it also provides a numerical solution to obtain the area weights and reduce the error that results for a nearly uniform distribution of points on a sphere. The method incorporates vector spherical harmonics to impose several modes of forcing on the surface of a sphere. The analytical force and torque resultants which can be readily obtained are made to match approximate results acquired by numerical integration using the corresponding area weights. It was shown that the numerical error is reduced by several orders of magnitudes when using this technique to calculate the area weights, rather than assuming that the markers on the sphere's surface are equally spaced.

Several simulations were performed for varying Reynolds numbers. The results from the present algorithm were compared to numerical and analytical data from the literature. Calculation of hydrodynamic forces and torque on a fixed sphere as well as freely moving spheres in a channel flow was carried out for a uniform and shear ambient flow. The results showed good agreement with previously reported values. The simulations of a random cluster of monodisperse spherical particles in a pressure driven channel flow showed a $76.96 \%$ reduction in the number of Lagrangian markers. The drag calculated through the Lagrangian forcing has a small error of $0.135 \%$ in comparison with the exact value. 


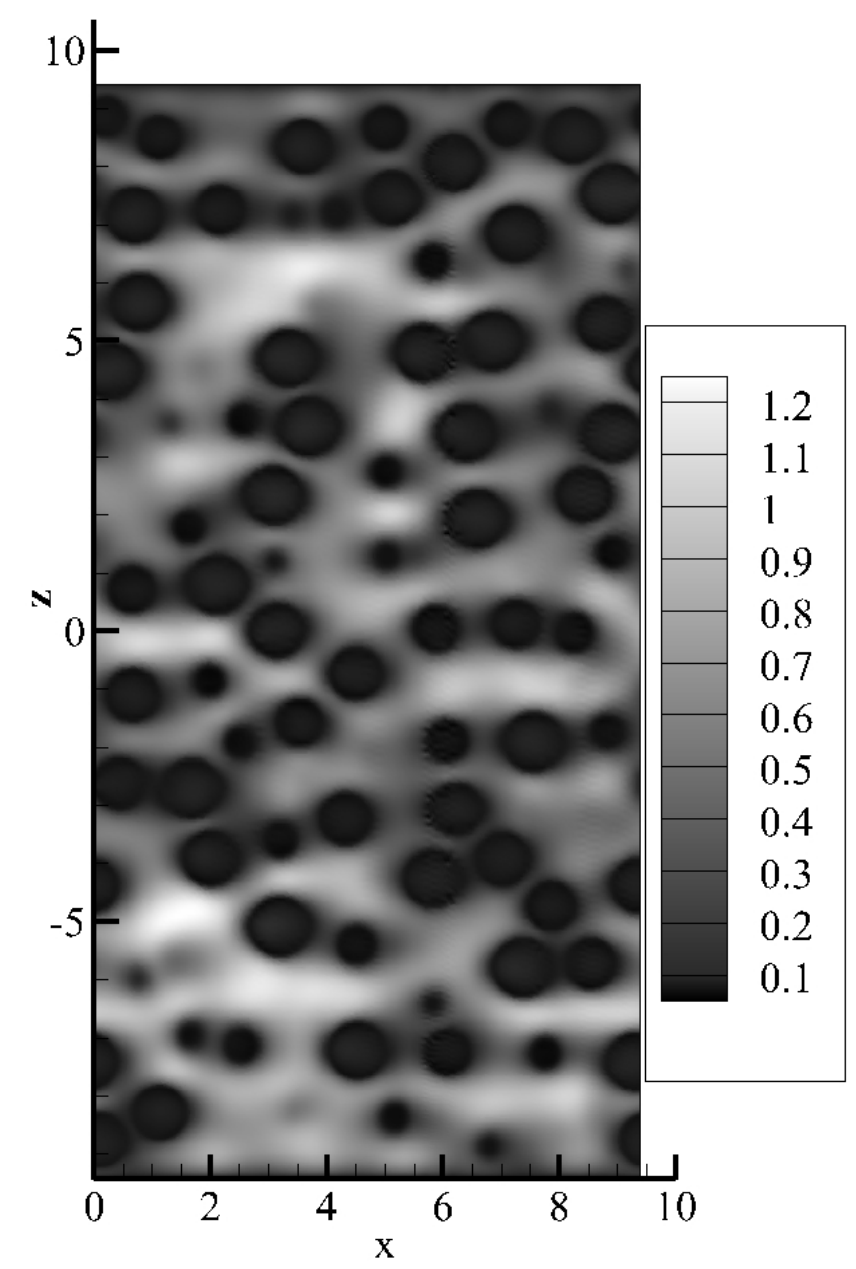

Figure 20. Contour plots of the streamwise velocity in the spanwise center plane.

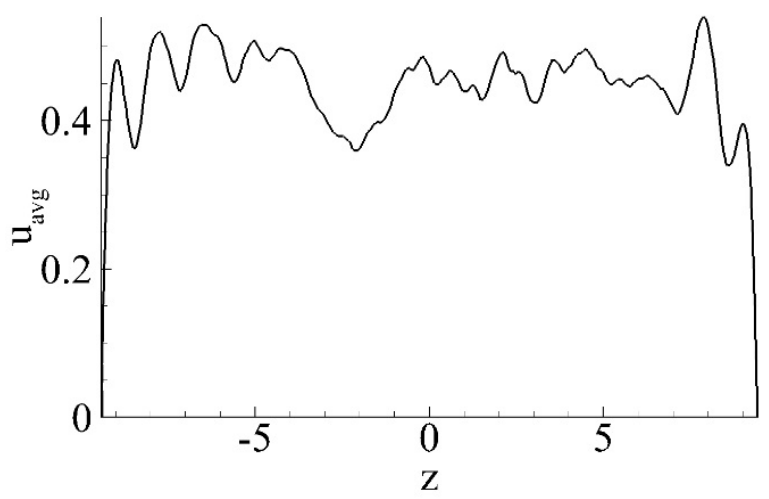

Figure 21. Streamwise planar averaged velocity as a function of the channel height. $R e_{b}=4.47$.

The method of the Lagrangian markers distribution in its current form can accommodate a nonuniform mesh in one direction, and the use of VSH to calculate the Lagrangian weights limits it to spherical particles. It will be the subject of future studies to be able to expand the formerly presented methods to accommodate arbitrary non-uniformities of the fluid grid and non-spherical particles. 


\section{Acknowledgments}

This work was supported by the National Science Foundation (NSF OISE-0968313) under Partnership for International Research and Education (PIRE) in Multiphase Flows at the University of Florida. Additionally, this work benefited from the U.S. Department of Energy, National Nuclear Security Administration, Advanced Simulation and Computing Program, as a Cooperative Agreement to the University of Florida under the Predictive Science Academic Alliance Program, under Contract No. DE-NA0002378.

\section{Appendix A.}

\section{A.1 Vector Spherical Harmonics Forces Surface Integration}

In Section 5.1, a vector spherical harmonic representation of tractional force on a spherical surface was defined. A three mode multipole expansion (for $I=1$ ) is rewritten here for convenience,

$$
\begin{aligned}
\mathbf{E}=( & \left.F_{00, r c}+F_{10, r c} \cos \theta-\sin \theta\left(F_{11, r c} \cos \phi+F_{11, r s} \sin \phi\right)\right) \hat{r} \\
& +\left(-F_{10, \Psi c} \sin \theta-F_{11, \Psi c} \cos \theta \cos \phi-F_{11, \Psi s} \cos \theta \sin \phi+F_{11, \Phi c} \sin \phi-F_{11, \Phi s} \cos \phi\right) \hat{\theta} \\
& +\left(F_{10, \Phi c} \sin \theta+F_{11, \Psi c} \sin \phi-F_{11, \Psi s} \cos \theta+F_{11, \Phi c} \cos \theta \cos \phi+F_{11, \Phi s} \cos \theta \sin \phi\right) \hat{\phi}
\end{aligned}
$$

The surface integration of $\mathbf{E}$ is performed using,

$$
\oiint_{S} \mathbf{E} d \theta d \phi=\int_{0}^{2 \pi} \int_{0}^{\pi} E_{m} \operatorname{Proj}(m, n)\left(r^{2} \sin \theta\right) d \theta d \phi
$$

where indices $m$ and $n$ represent the spherical and Cartesian coordinates respectively. $\operatorname{Proj}(m, n)$ is the term that would project the $m^{\text {th }}$ spherical coordinate to the $n^{\text {th }}$ Cartesian coordinate and is given in Table A1. Table A2 lists the contribution of the three spherical components of $\mathbf{E}$ in the three Cartesian directions, after being evaluated through equation (A.2).

The moment vector on the sphere is obtained by,

$$
\oiint_{S} \mathbf{E} D d \theta d \phi=\int_{0}^{2 \pi} \int_{0}^{\pi} E_{m}\left(\vec{d} \times \vec{p}_{m}\right)\left(r^{2} \sin \theta\right) d \theta d \phi
$$

$\vec{d}$ is the moment arm vector,

$$
\vec{d}=r \sin \theta \cos \phi \vec{i}+r \sin \theta \sin \phi \vec{j}+r \cos \theta \vec{k},
$$

and $\vec{p}$ is the polar coordinates unit vector,

$$
\begin{aligned}
& \vec{p}_{r}=\hat{r}=\sin \theta \cos \phi \vec{i}+\sin \theta \sin \phi \vec{j}+\cos \theta \vec{k} \\
& \vec{p}_{\theta}=\hat{\theta}=\cos \theta \cos \phi \vec{i}+\cos \theta \sin \phi \vec{j}-\sin \theta \vec{k} \\
& \vec{p}_{\phi}=\hat{\phi}=-\sin \phi \vec{i}+\cos \phi \vec{j}+0 \vec{k} .
\end{aligned}
$$

The contributions of the radial, tangential and azimuthal directions of the force distribution to the moment vector are, 
Table A1. Values of Proj $(l, m)$

\begin{tabular}{c|ccc}
\hline$n$ & & & \\
\hline$r$ & $\sin \theta \cos \phi$ & $\sin \theta \sin \phi$ & $\cos \theta$ \\
$\theta$ & $\cos \theta \cos \phi$ & $\cos \theta \sin \phi$ & $-\sin \theta$ \\
& $-\sin \phi$ & $\cos \phi$ & 0 \\
\hline
\end{tabular}

Table A2. Values of surface integral of the components of $\boldsymbol{E}$ projected on the Cartesian directions using Eq. A.2

\begin{tabular}{llll}
\hline $\boldsymbol{E}$ & $x$-direction & $y$-direction & $z$-direction \\
\hline \hline$E_{r}$ & $-\frac{4}{3} \pi r^{2} F_{11, r c}$ & $-\frac{4}{3} \pi r^{2} F_{11, r s}$ & $\frac{4}{3} \pi r^{2} F_{10, r c}$ \\
$E_{\theta}$ & $-\frac{2}{3} \pi r^{2} F_{11, \Psi_{c}}$ & $-\frac{2}{3} \pi r^{2} F_{11, \Psi_{s}}$ & $\frac{8}{3} \pi r^{2} F_{10, \Psi_{c}}$ \\
& $-2 \pi r^{2} F_{11, \Psi_{c}}$ & $-2 \pi r^{2} F_{11, \Psi_{s}}$ & 0 \\
\hline
\end{tabular}

$$
\begin{aligned}
& \int_{0}^{2 \pi} \int_{0}^{\pi} E_{r}\left(\vec{d} \times \vec{p}_{r}\right)\left(r^{2} \sin \theta\right) d \theta d \phi=0 \vec{i}+0 \vec{j}+0 \vec{k} \\
& \int_{0}^{2 \pi} \int_{0}^{\pi} E_{\theta}\left(\vec{d} \times \vec{p}_{\theta}\right)\left(r^{2} \sin \theta\right) d \theta d \phi=-2 \pi r^{3} F_{11, \phi c} \vec{i}-2 \pi r^{3} F_{11, \phi s} \vec{j}+0 \vec{k} \\
& \int_{0}^{2 \pi} \int_{0}^{\pi} E_{\phi}\left(\vec{d} \times \vec{p}_{\phi}\right)\left(r^{2} \sin \theta\right) d \theta d \phi=-\frac{2}{3} \pi r^{3} F_{11, \phi c} \vec{i}-\frac{2}{3} \pi r^{3} F_{11, \phi s} \vec{j}+\frac{8}{3} \pi r^{3} F_{10, \phi s} \vec{k}
\end{aligned}
$$

The results from Table A2 and equation (A.6) constitute equations (5.7)-(5.8).

\section{A.2 Discrete Summation of the Forces and Moments}

In numerical evaluation of the net force and moment on the sphere, a discrete summation is used over the Lagrangian markers. Each point is weighted by its unique $A_{l}$. The projections terms in equations. (A.2) and (A.3) are the same for their discrete counterpart.

$$
\begin{aligned}
& \oiint_{S} \mathbf{E} d \theta d \phi=\sum_{l=1}^{N_{L}} E_{m} \operatorname{Proj}(m, n)\left(r^{2} \sin \theta\right) A_{l} \\
& \oiint_{S} \mathbf{E} D d \theta d \phi=\sum_{l=1}^{N_{L}} E_{m}\left(\vec{d} \times \vec{p}_{m}\right)\left(r^{2} \sin \theta\right) A_{l}
\end{aligned}
$$

By considering 1 parameter at a time $\left(F_{00, r c}, F_{10, \Psi_{c}}, F_{11, \Phi_{s}}, \ldots\right)$, a total of six equations, three from equation (A.7) and three from equation (A.8), can be obtained for each profile separately. Some of the profiles vanishes for certain directions, leading to the trivial solution only. Instead of having 60 equations, a total of 47 remain. Several of these equations are linearly dependent. The 47 equations are written in matrix form as explained in Section 5.2. A slightly modified "rref" built-in function in Matlab was used. The function uses Gauss Jordan elimination with partial pivoting. A tolerance of $1 \times 10^{-5}$ was specified. This leads to a reduced row echelon form of matrix $M$ which was defined in equation (5.18). The rank of $M$ turns out to be 9 . The 9 selected linearly independent equations are given in (5.19)-(5.27). 


\section{Reference}

Apte, Sourabh V., Mathieu Martin, and Neelesh A. Patankar. "A numerical method for fully resolved simulation (FRS) of rigid particle-flow interactions in complex flows." Journal of Computational Physics 228.8 (2009): 2712-2738.

N.N. Andreev, "An extremal property of the icosahedron", East J. Approximation, 2(4) (1996), 459-462, MR 97m:52022, Zbl 0877.51021

Bagchi, P., and S. Balachandar. "Effect of free rotation on the motion of a solid sphere in linear shear flow at moderate Re." Physics of Fluids (1994-present)14.8 (2002): 2719-2737.

Barrera, R. G., G. A. Estevez, and J. Giraldo. "Vector spherical harmonics and their application to magnetostatics." European Journal of Physics 6.4 (1985): 287.

Canuto, C., Hussaini, M. Y., Quarteroni, A., \& Zang, T. A. (1988). "Spectral methods in fluid dynamics." No. CMCS-BOOK-2009021. Springer.

Cortese, Thomas A., and S. Balachandar. "High performance spectral simulation of turbulent flows in massively parallel machines with distributed memory." International Journal of High Performance Computing Applications 9.3 (1995): 187-204.

Dorai, F., Teixeira, C. M., Rolland, M., Climent, E., Marcoux, M., \& Wachs, A. (2015). "Fully-resolved simulations of the flow through a packed bed of cylinders: Effect of size distribution." Chemical Engineering Science.

Favier, Julien, Alistair Revell, and Alfredo Pinelli. "A Lattice Boltzmann-Immersed Boundary method to simulate the fluid interaction with moving and slender flexible objects." Journal of Computational Physics 261 (2014): 145-161.

Feng, Zhi-Gang, and Efstathios E. Michaelides. "Heat transfer in particulate flows with direct numerical simulation (DNS)." International Journal of Heat and Mass Transfer 52.3 (2009): 777-786.

Garg, R., Tenneti, S., Mohd-Yusof, J., Subramaniam, S., 2011. Direct numerical simulation of gas-solids flow based on the immersed boundary method. In: Pannala, S., Syamlal, M., O’Brien, T.J. (Eds.), Computational Gas-Solids Flows and Reacting Systems: Theory, Methods and Practice. IGI Global, pp. 245-276.

Gilmanov, Anvar, and Fotis Sotiropoulos. "A hybrid Cartesian/immersed boundary method for simulating flows with 3D, geometrically complex, moving bodies." Journal of Computational Physics 207.2 (2005): 457-492.

Hirt, C. W., Anthony A. Amsden, and J. L. Cook. "An arbitrary Lagrangian-Eulerian computing method for all flow speeds." Journal of Computational Physics 14.3 (1974): 227-253.

Kevin Brown. "Min-Energy Configurations of Electrons on a Sphere". Retrieved 2014-05-01.

Kidanemariam, Aman G., and Markus Uhlmann. "Interface-resolved direct numerical simulation of the erosion of a sediment bed sheared by laminar channel flow." International Journal of Multiphase Flow 67 (2014): 174-188.

Klug, A., W. Longley, and R. Leberman. "Arrangement of protein subunits and the distribution of nucleic acid in turnip yellow mosaic virus: I. X-ray diffraction studies."Journal of molecular biology 15.1 (1966): 315-IN16.

Kurose, Ryoichi, and Satoru Komori. "Drag and lift forces on a rotating sphere in a linear shear flow." Journal of Fluid Mechanics 384 (1999): 183-206.

Lee, Hyungoo, and S. Balachandar. "Drag and lift forces on a spherical particle moving on a wall in a shear flow at finite Re." Journal of Fluid Mechanics 657 (2010): 89-125.

Lee, Hyungoo, Man Yeong Ha, and S. Balachandar. "Rolling/sliding of a particle on a flat wall in a linear shear flow at finite Re." International Journal of Multiphase Flow 37.2 (2011): 108-124.

McLaughlin, John B. "Inertial migration of a small sphere in linear shear flows."Journal of Fluid Mechanics 224 (1991): $261-274$.

Mehrabadi, M., Tenneti, S., Garg, R., \& Subramaniam, S. (2015). Pseudo-turbulent gas-phase velocity fluctuations in homogeneous gas-solid flow: fixed particle assemblies and freely evolving suspensions. Journal of Fluid Mechanics, 770, 210-246. 
Mei, R. "An approximate expression for the shear lift force on a spherical particle at finite Reynolds number." International Journal of Multiphase Flow 18.1 (1992): 145-147.

Mittal, Rajat, and Gianluca Iaccarino. "Immersed boundary methods." Annu. Rev. Fluid Mech. 37 (2005): 239-261.

Peskin, Charles S. "Flow patterns around heart valves: a numerical method."Journal of Computational Physics 10.2 (1972): $252-271$.

Pinelli, A., Naqavi, I. Z., Piomelli, U., \& Favier, J. (2010). "Immersed-boundary methods for general finite-difference and finitevolume Navier-Stokes solvers." Journal of Computational Physics, 229(24), 9073-9091.

Roma, Alexandre M., Charles S. Peskin, and Marsha J. Berger. "An adaptive version of the immersed boundary method." Journal of computational physics 153.2 (1999): 509-534.

Saff, Edward B., and A. BJ Kuijlaars. "Distributing many points on a sphere." The Mathematical Intelligencer 19.1 (1997): 5-11.

Saffman, PGt. "The lift on a small sphere in a slow shear flow." Journal of Fluid Mechanics 22.02 (1965): 385-400.

Schiller, L., and A. Naumann. "Über die grundlegenden Berechnungen bei der Schwerkraftaufbereitung." Ver. Deut. Ing 77 (1933): 318-320.

Tenneti, S., Garg, R., \& Subramaniam, S. (2011). Drag law for monodisperse gas-solid systems using particle-resolved direct numerical simulation of flow past fixed assemblies of spheres. International journal of multiphase flow, 37(9), $1072-1092$.

Thomson, J. J. "On the Structure of the Atom: an Investigation of the Stability and Periods of Oscillation of a number of Corpuscles arranged at equal intervals around the Circumference of a Circle; with Application of the Results to the Theory of Atomic Structure", Philosophical MagazineSeries 6, Volume 7, Number 39, pp. 237-265, March 1904

Uhlmann, Markus. "An immersed boundary method with direct forcing for the simulation of particulate flows." Journal of Computational Physics 209.2 (2005): 448-476.

Uhlmann, Markus, and Todor Doychev. "Sedimentation of a dilute suspension of rigid spheres at intermediate Galileo numbers: the effect of clustering upon the particle motion." Journal of Fluid Mechanics 752 (2014): 310-348.

V.A. Yudin, "The minimum of potential energy of a system of point charges", Discretnaya Matematika 4(2) (1992), 115-121 (in Russian); Discrete Math. Appl., 3(1) (1993), 75-81

Van der Hoef, M. A., van Sint Annaland, M., Deen, N. G., \& Kuipers, J. A. M. (2008). "Numerical simulation of dense gas-solid fluidized beds: A multiscale modeling strategy". Annu. Rev. Fluid Mech., 40, 47-70.

Zeng, L., Balachandar, S., Fischer, P., \& Najjar, F. (2008). "Interactions of a stationary finite-sized particle with wall turbulence." Journal of Fluid Mechanics,594, 271-305.

Zeng, L., Najjar, F., Balachandar, S., \& Fischer, P. (2009). "Forces on a finite-sized particle located close to a wall in a linear shear flow." Physics of Fluids (1994-present), 21(3), 033302.

Zhang, L., Gerstenberger, A., Wang, X., \& Liu, W. K. (2004). "Immersed finite element method." Computer Methods in Applied Mechanics and Engineering, 193(21), 2051-2067. 